\title{
SBAS DFMC service for Road Transport: Positioning and integrity monitoring with a new weighting model
}

\author{
K. Wang • A. El-Mowafy · C. Rizos • J. Wang
}

the date of receipt and acceptance should be inserted later

\begin{abstract}
In 2017, the new generation Satellite-Based Augmentation System (SBAS) test-bed was initiated by Australia and New Zealand, which supports the dual-frequency multi-constellation (DFMC) positioning with both GPS and Galileo signals. This new SBAS DFMC service allows the elimination of the first-order term of the ionospheric delays, and extends the service area to the entire footprint of the geostationary satellite. In addition to the satellite clock and orbital corrections, the integrity information is also broadcast by the SBAS satellite to users, so that protection levels can be computed to bound the positioning errors with a pre-defined probability of hazardous misleading information. Different from the aeronautical applications, the ground-based applications for road transport may suffer from new problems in different measurement environments, e.g., complicated multipath behaviours and frequent filter re-initialisations during positioning in urban areas. A new weighting model allowing different impacts of the elevation angles, the signal-to-noise-ratios and the smoothing time after re-initialisations is proposed and compared with the traditional elevation-dependent weighting model. The model is applied to the carrier-smoothed code measurements in different environments, i.e., the open-sky scenario, the suburban scenario and the urban scenario. It is found that the new weighting model effectively de-weights the large residuals in the suburban and the urban scenarios, where the mean values and the standard deviations of the overbounding excess-mass cumulative density function can be significantly reduced for the combined weighted noise and multipath. Using $1 \mathrm{~Hz}$ GNSS observations measured in these three measurement environments, the horizontal positioning errors (HPEs) and the horizontal protection levels (HPLs) are computed for different filter smoothing windows. Applying the new weighting model, significant reduction can be observed in the mean HPLs in the suburban and urban scenarios. Among them, the reduction in the HPLs have reached about $35-40 \%$ in the suburban scenario. The mean absolute HPEs are also reduced by about $10 \%$ in the urban scenario. However, when under the open-sky scenario, the traditional elevation-dependent weighting model is sufficient for the positioning and integrity monitoring using the SBAS DFMC service.
\end{abstract}

Keywords Global Navigation Satellite System (GNSS) - Satellite-Based Augmentation System (SBAS) · Dual-Frequency Multi-Constellation (DFMC) · Integrity Monitoring (IM) · Weighting model · Signal-toNoise-Ratio (SNR)

K. Wang

School of Earth and Planetary Sciences, Curtin University, Perth, Australia

E-mail: kan.wang@curtin.edu.au

A. El-Mowafy

School of Earth and Planetary Sciences, Curtin University, Perth, Australia

E-mail: A.El-Mowafy@curtin.edu.au

C. Rizos

School of Civil and Environmental Engineering, UNSW, Sydney, Australia

E-mail: c.rizos@unsw.edu.au

J. Wang

School of Civil and Environmental Engineering, UNSW, Sydney, Australia

E-mail: jinling.wang@unsw.edu.au 


\section{Introduction}

The satellite-based augmentation system (SBAS) is a widely-used GNSS-augmented service, which improves the accuracy, availability and reliability of the positioning service by broadcasting precise satellite-related corrections, integrity information, and extra ranging signals from geostationary (GEO) satellites to users in real time. During the last decades, different SBAS were put into usage and are continuously providing wide-area or regional augmentation to the user positioning. Examples are the Wide Area Augmentation System (WAAS) of the USA, the European Geostationary Navigation Overlay Service (EGNOS), the Multi-functional Satellite Augmentation System (MSAS) of Japan, and the GPS Aided Geo Augmented Navigation (GAGAN) system operated by India. These SBASs were mainly developed for aviation, and hence fulfil the corresponding aviation requirements (RTCA 2016). An overview can be found in Choy et al. (2017).

Making use of the raw GNSS measurements collected from a ground reference network, the traditional SBAS service transmits satellite-related corrections, integrity information, and extra ranging signals via the L1 frequency at $1575.42 \mathrm{MHz}$ to users, supporting the single-frequency single-constellation positioning service (RTCA 2016). The service area is in such a way limited by the ionospheric corrections broadcast to the users, or in other words, limited by the ground network processing the ionospheric delays. The positioning accuracy is also sensitive to the accuracy of the ionospheric corrections. Studies were performed to investigate different variants of the ionospheric corrections and their impacts on the accuracy and integrity of the SBAS L1 service (Grunwald et al. 2016). Making use of two frequencies instead, the first-order term of the ionospheric delays, which occupies about $99 \%$ of the total ionospheric effect (Hoque and Jakowski 2012), can be eliminated by forming the ionosphere-free (IF) linear combination. Without needing to broadcast the ionospheric corrections, the service area of the SBAS is extended to the entire footprint of the GEO satellite.

Moving to the next generation of SBAS by many existing systems like WAAS and EGNOS, a second frequency, i.e., the L5 frequency at $1176.45 \mathrm{MHz}$, is utilised for signal transmission from the SBAS satellites. The new service tends to support dual-frequency multi-constellation (DFMC) positioning service, which does not only allow the removal of the first-order ionospheric delays and extend the service area as mentioned before, but is also better suitable for the safety-of-life applications due to be stronger interference resistance of the L5 signals with other signals (Choy et al. 2017). The inclusion of other GNSSs in addition to GPS, at the same time, allows for an increasing number of usable satellites and thus improved measurement geometry and positioning precision. In early 2017, a two-year SBAS test-bed was initiated by Australia and New Zealand, and operated as a test phase for a fully operational second-generation SBAS service in the near future by the Geoscience Australia (GA) and the Land Information New Zealand (LINZ), and managed by the FrontierSI. The test-bed was running in collaboration with the industrial partners such as the GMV providing the master station and the server, the Lockheed Martin Space Systems Company which hosts the master station and supports the system integration and the uplink system, and the Inmarsat providing the navigation payload on the GEO satellite of the test-bed, i.e., the Inmarsat-4F1 with a pseudo-random noise (PRN) of 122 (FrontierSI 2017). In addition to the L1 service, the GEO satellite transmits SBAS DFMC corrections and integrity information through L5 to support the user positioning with GPS L1/L5 and Galileo E1/E5a measurements. Furthermore, precise point positioning (PPP) corrections are also sent to users via L1 and L5 to support the PPP service using dual-frequency GPS and GPS/Galileo signals, respectively. Due to the limited number of the GPS IIF satellites transmitting L5 signals at the moment, the GPS L2 signals were used instead to supply a better geometry and positioning precision within the test-bed before 2019, and now switched to L1/L5. With an increasing number of the GPS IIF and even III satellites launched in the next ten years, and with the better signal precision of the L5 code measurements (Nadarajah et al. 2016), improved behaviours can be expected in the future.

The integrity monitoring (IM) is a vital part of the SBAS positioning service, as it is originally designed for aviation as a safety-related application. IM improves the reliability of the positioning by ensuring that its errors are within the protection levels under a pre-defined probability of hazardous misleading information (PHMI). Warnings are sent to the users within the maximum allowable elapsed time, referred to as timeto-alert, when the protection level exceeds the alert limit and a positioning failure is recognized. In IM the positioning results are considered available when the protection level is lower than the alert limit. Making use of the DFMC GNSS signals, the Receiver Autonomous Integrity Monitoring (RAIM) algorithm designed to provide horizontal guidance for aeronautical operations (Lee 1986; Parkinson and Axelrad 1988) was extended to an advanced RAIM (ARAIM) algorithm, which is applied for positioning with vertical 
guidance in addition. The algorithms of the ARAIM, including the fault detection and exclusion (FDE) procedures and the computation of protection levels, were extensively studied in the last ten years using DFMC data (Blanch et al. 2012, 2013; ARAIM 2016). El-Mowafy (2017) shows improvement of ARAIM using triple-frequency data. The algorithms were also applied in ground-based applications using PPP and real-time kinematic (RTK) positioning integrated with inertial measurement unit (IMU) and odometers (El-Mowafy and Kubo 2017, 2018; Gunning et al. 2018).

The integrity and accuracy of the SBAS L1 service and the planned SBAS DFMC service were studied for WAAS and EGNOS over the last ten years. Grunwald et al. (2016) performed the accuracy and integrity analysis of the EGNOS SBAS L1 service having different calculation variants dealing with the ionospheric corrections. Wanner et al. (2008) presented analysis verifying the WAAS vertical accuracy under different ionosphere conditions. Walter et al. (2010a,b) demonstrated reduced protection levels, higher availability and improved coverage for the possible dual-frequency WAAS. The minimum operational performance standard (MOPS) of the SBAS L5 service augmenting GPS and/or Galileo in dual-frequency mode is strictly defined in EUROCAE (2019) for aeronautical applications.

SBAS was originally developed for aviation with good satellite visibility and simple multipath conditions. However, the positioning and IM for ground-based applications in road transport, which involve a large sector of the massive users, face new problems like different and more complicated multipath environments. In urban areas, large code multipath could exist under relatively high elevation angles, and subsequent cycle slips in phase measurements may lead to frequent re-initialisations of the carrier-phase smoothing filter. This could make the traditional elevation-dependent weighting model insufficient for applications in such environments. Already from 20 years ago, the signal-to-noise-ratios (SNRs) were utilised to calculate the variance of the GPS observations (Hartinger and Brunner 1999; Wieser and Brunner 2000). Considering both the influences of the elevation angles and the SNRs, another model was developed by Tay and Marais (2013) for GPS pseudorange observations in urban canyons. Vehicles in road transport and pedestrian navigation are potential users of this technology.

In this contribution, based on the carrier-smoothed code measurements used for the SBAS DFMC service, a new weighting model aiming at improving results is developed allowing impacts of the elevation angles, the SNRs and the smoothing time in different measurement environments, i.e., the open-sky scenario, the suburban scenario and the urban scenario. Based on the weighting effects optimised for the combined noise and multipath in these environments, the overbounding excess-mass cumulative distribution functions (CDFs) are searched for the weighted noise and multipath. Applying both the new model and the traditional elevation-dependent weighting model, the horizontal positioning errors (HPEs) and horizontal protection levels (HPLs) are computed and compared using real data collected in different environments and for different smoothing windows in the filter. The integrity performance is afterwards evaluated using the two models and compared based on the HPEs, HPLs and an assumed horizontal alert limit (HAL). Different from Wang et al. (2020) that utilises the elevation angles and the SNRs to weight the double-differenced GPS L1 and L2 code and phase observations in the RTK positioning, in this contribution, the filter smoothing time is introduced, and its impact is adjusted along with the impacts of the elevation angles and the SNRs to weight the IF carrier-smoothed code observations for the DFMC SBAS positioning under different measurement scenarios. The algorithm for integrity monitoring is modified at the receiver-end based on the new standards EUROCAE (2019), and overbounding excess-mass CDFs (EMCs) are searched for the combined weighted noise and multipath for both the GPS and Galileo satellites.

The paper starts with the signal analysis, which includes the proposal of the new weighting model, the search of the model coefficients for different smoothing windows and environments, and presents the overbounding EMCs for the combined weighted noise and multipath. The observation model for the SBAS DFMC positioning is then described, followed by the procedure of FDE and the principles of computing the HPLs. The real data and the SBAS message broadcast via L5 within the SBAS test-bed are then utilised to compute the SBAS DFMC HPEs and HPLs. The scenario is adapted for different environments, smoothing windows and weighting functions, followed by a comparison of the results. The conclusion is given at the end.

\section{Signal analysis}

In the SBAS DFMC processing using dual-frequency GPS signals on L1/L2, and Galileo signals on E1/E5a, a filter is utilised to perform the IF code-carrier smoothing. The IF carrier-smoothed code observations 
from satellite $s$ at $t_{i}$, denoted by $\tilde{p}_{I F}^{s}\left(t_{i}\right)$, can be computed with a Hatch filter as (Hatch 1982):

$$
\tilde{p}_{I F}^{s}\left(t_{i}\right)=\frac{1}{n} p_{I F}^{s}\left(t_{i}\right)+\frac{n-1}{n}\left(\tilde{p}_{I F}^{s}\left(t_{i-1}\right)+\phi_{I F}^{s}\left(t_{i}\right)-\phi_{I F}^{s}\left(t_{i-1}\right)\right) \quad\left\{\begin{array}{l}
n=i, \forall i<N \\
n=N, \forall i \geq N
\end{array}\right.
$$

where the IF linear combination of the raw phase and code observations from satellite $s$ are represented by $p_{I F}^{s}$ and $\phi_{I F}^{s}$, respectively. The terms $n$ and $N$ denote the number of the smoothing epochs since initialisation, and a pre-defined smoothing window, respectively. After reaching the $N$-th epoch, $n$ is equal to $N$. For quality control, a rough code outlier detection is performed comparing the IF raw code observation $p_{I F}^{s}\left(t_{i}\right)$ and the projected observation $p_{\text {proj }}^{s}\left(t_{i}\right)=\tilde{p}_{I F}^{s}\left(t_{i-1}\right)+\left(\phi_{I F}^{s}\left(t_{i}\right)-\phi_{I F}^{s}\left(t_{i-1}\right)\right)$. Code outliers would be identified and the code observation is excluded when the difference between $p_{I F}^{s}\left(t_{i}\right)$ and $p_{\text {proj }}^{s}\left(t_{i}\right)$ exceeds $10 \mathrm{~m}$ (EUROCAE 2019). The filter is re-initialised whenever a cycle slip is detected in the phase observations, or when a gap of unavailable and unusable observations is experienced longer than a pre-defined time interval, here e.g. $6 \mathrm{~s}$ as the time-to-alert for the localizer performance with vertical guidance (LPV) approach in aviation. In this contribution, a sliding time window observing $N$ epochs of data is used when the number of smoothing epochs reaches $N$. Eq. 1 can thus be reformulated as (Le and Teunissen 2008):

$$
\tilde{p}_{I F}^{s}\left(t_{i}\right)=\phi_{I F}^{s}\left(t_{i}\right)+\frac{1}{n} \sum_{j=i-n+1}^{i} p_{I F}^{s}\left(t_{j}\right)-\frac{1}{n} \sum_{j=i-n+1}^{i} \phi_{I F}^{s}\left(t_{j}\right), \quad\left\{\begin{array}{l}
n=i, \forall i<N \\
n=N, \forall i \geq N
\end{array}\right.
$$

To show the effects of the carrier-smoothing, the geometric distance, the tropospheric delays, and the receiver and satellite clock errors are removed first from $\tilde{p}_{I F}^{s}$ by subtracting the IF phase observations $\phi_{I F}^{s}$. To eliminate the ambiguities and the major part of the hardware biases, a mean value is then removed from the residuals as follows:

$$
\Delta \tilde{p}^{s}\left(t_{i}\right)=\Delta \tilde{p}_{I F}^{s}\left(t_{i}\right)=\tilde{p}_{I F}^{s}\left(t_{i}\right)-\phi_{I F}^{s}\left(t_{i}\right)-\frac{\sum_{k=c_{1}}^{c_{a}}\left(\tilde{p}_{I F}^{s}\left(t_{k}\right)-\phi_{I F}^{s}\left(t_{k}\right)\right)}{c_{a}-c_{1}+1}
$$

where $c_{1}$ and $c_{a}$ denote the starting and the ending epochs of the time period with constant ambiguity, respectively. Ignoring the small noise and multipath of the phase observations, the term $\Delta \tilde{p}^{s}$ consists mainly of the noise and multipath in the smoothed code observations. It is worth noting that by using Eq. 3, the mean multipath effects in the smoothed observations are also removed. Here as an approximation, they are assumed to be small and are not further considered in the data analysis. In this contribution, $\Delta \tilde{p}^{s}$ larger than $10 \mathrm{~m}$ is considered to experience very large multipath or non-line-of-sight (NLOS) errors. They are excluded as outliers from the signal analysis, as it is assumed that the outlier detection procedure would exclude these large outliers in the data pre-processing phase. For simplification, the subscript IF in $\Delta \tilde{p}_{I F}^{s}$ will be dropped in the remainder of the paper, i.e., $\Delta \tilde{p}_{I F}^{s}$ will be denoted as $\Delta \tilde{p}^{s}$, likewise for $\Delta p^{s}$ in the next paragraph.

For reason of comparison, the raw IF code residuals are also computed by subtracting the raw IF phase measurements $\phi_{I F}^{s}$ from the raw code observations $p_{I F}^{s}$. The mean values are then removed from the residuals afterwards, approximating the raw code noise and multipath with:

$$
\Delta p^{s}\left(t_{i}\right)=\Delta p_{I F}^{s}\left(t_{i}\right)=p_{I F}^{s}\left(t_{i}\right)-\phi_{I F}^{s}\left(t_{i}\right)-\frac{\sum_{k=c_{1}}^{c_{a}}\left(p_{I F}^{s}\left(t_{k}\right)-\phi_{I F}^{s}\left(t_{k}\right)\right)}{c_{a}-c_{1}+1}
$$

The combined noise and multipath in the raw and smoothed IF code observations, i.e., the terms $\Delta p^{s}$ (Eq. 4) and $\Delta \tilde{p}^{s}$ (Eq. 3) are shown in the top panels of Figure 1, as an example, for the GPS satellite G27. The data of the static station FTDN in Sydney under the open-sky scenario (panel a), and those collected from a moving vehicle in the urban areas of Sydney on April 24, 2018 were used for the plots. Compared to the open-sky scenario, the smoothed measurements in the urban scenario experienced more re-initialisations due to the subsequent cycle slips and data gaps. Low SNRs frequently appear during the experiment, and large smoothed residuals are often observed at the beginning of the frequent re-initialisations. 

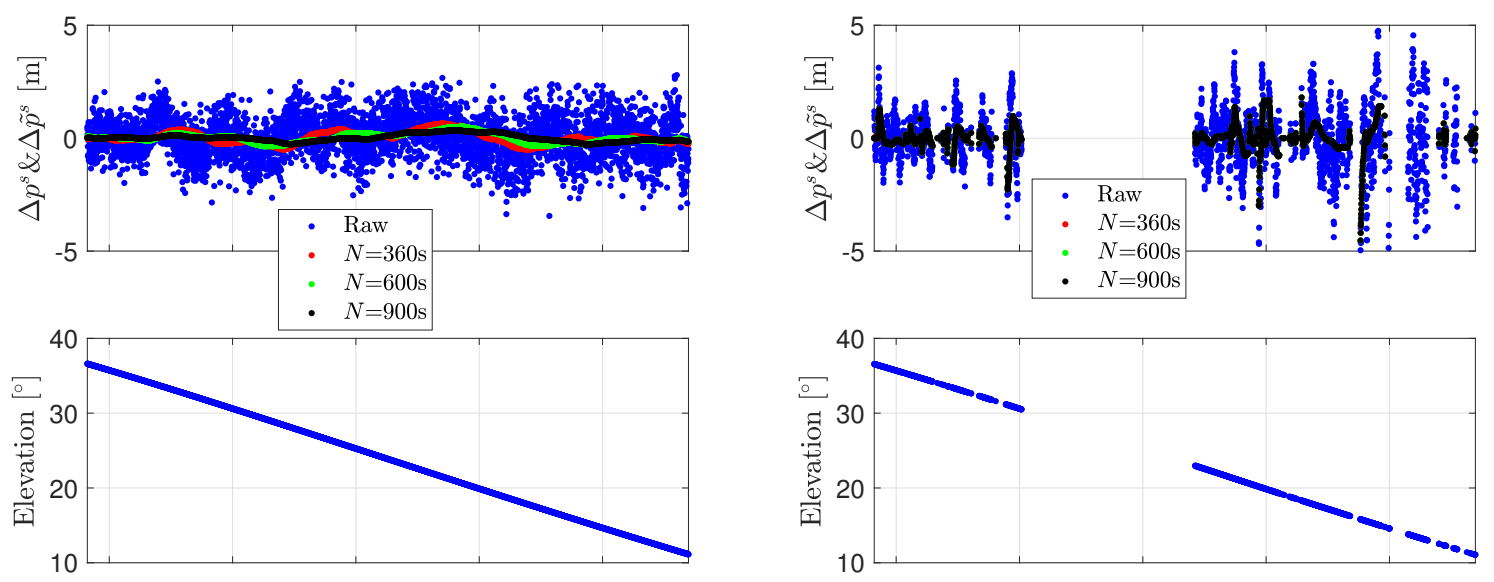

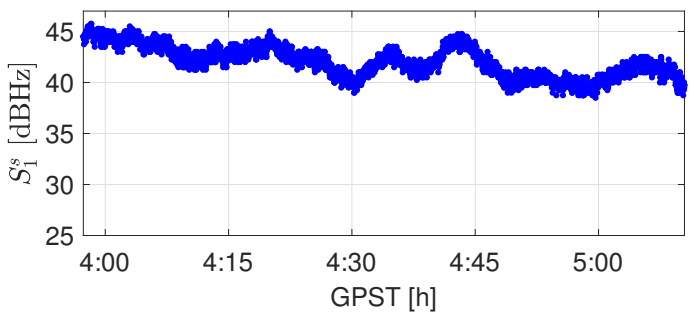

(a) Open-sky scenario

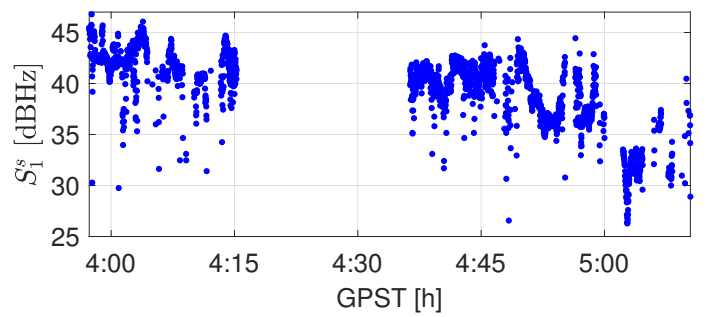

(b) Urban scenario

Fig. 1 Combined noise and multipath in the raw $\left(\Delta p^{s}\right)$ and carrier-smoothed $\left(\Delta \tilde{p}^{s}\right)$ IF code observations (top), as well as the corresponding elevation angles (middle) and SNRs on L1 (bottom). The data of G27 from the static station FTDN in Sydney under the open-sky scenario (a), and the data collected from a moving vehicle in the urban scenario of Sydney (b) on April 24, 2018 were used. GPST denotes the GPS time.

\subsection{Weighting model}

Traditionally, the elevation-dependent weighting function is used to weight the combined noise and multipath during the position estimation process through the least-squares adjustment (Euler and Goad 1991; Malkin Z 2008; Teunissen and de Bakker 2012; El-Mowafy 2015). In urban areas, however, the elevationdependent modelling may not be sufficient to describe the variation of the multipath anymore. Large multipath may still appear at relatively high elevation angles, and the smoothed code observations are facing an increasing number of the filter re-initialisations in urban areas due to the cycle slips and data gaps (Figure 1b). As will be shown later in this section, the amplitude of the smoothed noise and the multipath is strongly correlated with the smoothing time after each re-initialisation. Compared to the open-sky scenario, the percentage of the time epochs with low SNRs and short smoothing time has significantly increased in the urban scenario.

Figure 2 shows the relationships between $\Delta \tilde{p}^{s}$ (Eq. 3) and the elevation angles $\theta^{s}$ (panel a), and the smoothing epochs $n$ (panel b) in different measurement environments for the smoothing window $N=900$. In Figure 2, using all available GPS and Galileo satellites, the data collected from the static station UWA0 on September 13, 2018 was used for the plots in the top panels for the open-sky scenario. For the middle and bottom panels, the situations in the suburban and urban scenarios are illustrated using data collected from moving vehicles in different time periods from April to June 2018 in Wollongong and Sydney, Australia. Here the suburban scenario is characterised by a medium-level of satellite visibility and environment complexity, whereas the urban scenario is characterised by a relatively low satellite visibility and high environment complexity. Note that the measurements in the Central Business District (CBD) with the highest density are not included in this study. From Figure 2a it can be observed that the strength of the correlation between the elevation angles and the combined noise and multipath generally decreases with the increasing complexity of the measurement environments. In the urban scenario, i.e., the bottom panel of Figure 2a, 

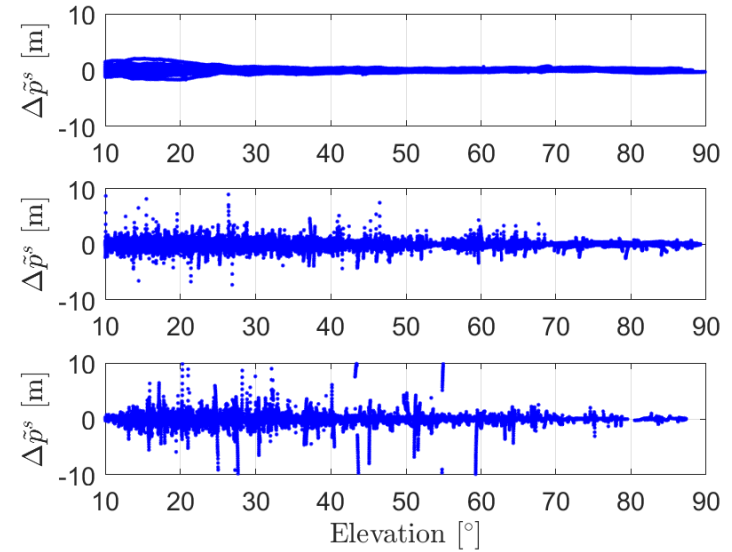

(a) $\Delta \tilde{p}^{s} \sim$ Elevation
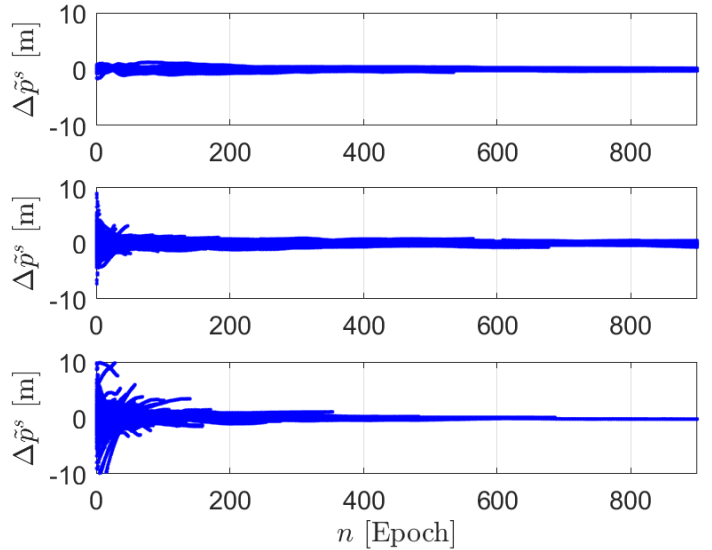

(b) $\Delta \tilde{p}^{s} \sim n$

Fig. 2 Relationships between the $\Delta \tilde{p}^{s}$ and: (a) the elevation angles and (b) the number of the smoothing epochs $n$ (with $n<N)$ for $N=900$. The data collected from all usable satellites in the open-sky scenario (top), in the suburban scenario (middle) and in the urban scenario (bottom) were used for the plots. The data of all available GPS and Galileo satellites from the station UWA0 on September 13, 2018 were used for the open-sky scenario, and data collected from moving vehicles in different time periods from April to June, 2018 were used for the suburban and urban scenarios

large multipath effects are also observable at relatively high elevation angles. The corresponding correlation coefficient varies from -0.24 in the open-sky scenario to -0.16 in the urban scenario. Figure $2 \mathrm{~b}$ shows the correlation between the smoothing time $n$ and the $\Delta \tilde{p}^{s}$. Among all the measurements, samples with $n<50$ have increased from below $0.1 \%$ in the open-sky scenario to above $40 \%$ in the urban scenario. With the increasing multipath and number of the filter re-initialisations from the open-sky to the urban scenarios, increasing strength of the correlation can be observed between $\Delta \tilde{p}^{s}$ and the smoothing epochs $n$ from the top to the bottom panels. The corresponding correlation coefficient, which is calculated as the sample Pearson correlation coefficient, varies from -0.05 in the open-sky scenario to -0.17 in the urban scenario. A pure elevation-dependent weighting model is thus not sufficient for ground-based applications in complicated environments using the SBAS DFMC service. Considering the smoothing time is essential to deliver a better weighting function.

It should be noted that in aviation, the DFMC SBAS only makes use of the well smoothed observations, i.e., with $n=N$. For ground-based applications under complicated measurement environment, however, the observations with $n<N$ occupies a large amount of data. Sacrificing these data would lead to worse measurement geometry under already limited satellite visibility. For example, Figure 3 shows the percentages of the data that would be lost when not using data with smoothing epochs smaller than or equal to a pre-defined number of epochs $M$, where $M$ is smaller than the smoothing window $N$. As a result, this study proposed to use these data, but appropriately de-weight them as discussed later in this section.

As mentioned before, the SNR is also a factor correlated with the noise and multipath. As the SNRs within the smoothing time may significantly change, the average SNRs, denoted as $\bar{S}_{k}^{s}(k=u, v)$, is calculated with the mean signal variance based on a SNR-dependent weighting function, where $u$ and $v$ refer to the first and the second frequency used to form the IF combination:

$$
\sigma_{0, k, \text { raw }}^{2} \times\left(1+m_{k} \times 10^{-0.1 \times \bar{S}_{k}^{s}\left(t_{i}\right)}\right)=\frac{\sigma_{0, k, \text { raw }}^{2}}{n} \times \sum_{j=i-n+1}^{i}\left(1+m_{k} \times 10^{-0.1 \times S_{k}^{s}\left(t_{j}\right)}\right)
$$

where $m_{k}$ is a coefficient varying with the frequency $k$, and $\sigma_{0, k \text {,raw }}$ refers to the standard deviation of the raw observations on frequency $k$ at a SNR of $\infty$. The SNR-related weighting model is derived based on the SIGMA- $\epsilon$ model (Wieser and Brunner 2000) using the form $V+C \times 10^{-0.1 \times \mathrm{SNR}}$, where $V$ and $C$ are the model-selected coefficients. The $\bar{S}_{k}^{s}$ can thus be obtained with:

$$
\bar{S}_{k}^{s}\left(t_{i}\right)=-10 \times \log _{10}\left(\frac{1}{n} \times \sum_{j=i-n+1}^{i} 10^{-0.1 \times S_{k}^{s}\left(t_{j}\right)}\right)
$$




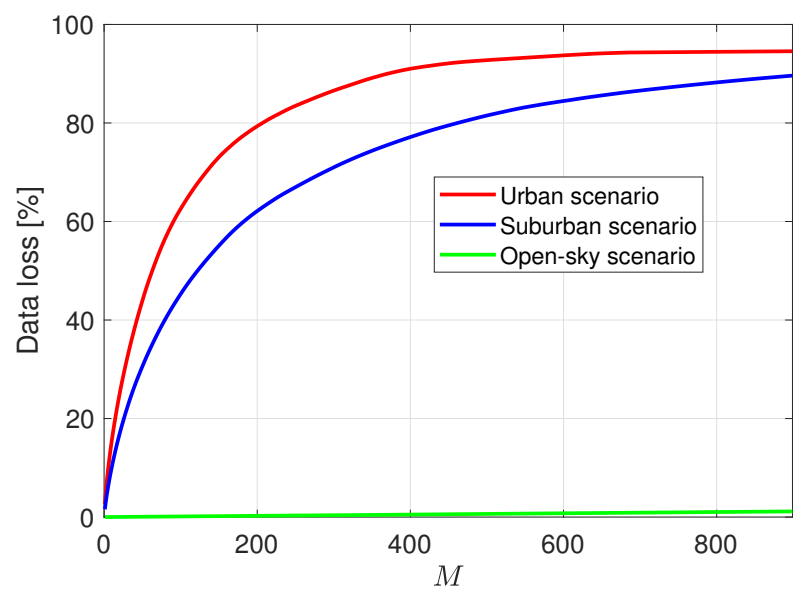

Fig. 3 Percentages of the data loss when excluding data having smoothing epochs smaller than or equal to $M(M<N)$. $N$ is set to 900 epochs for this plot
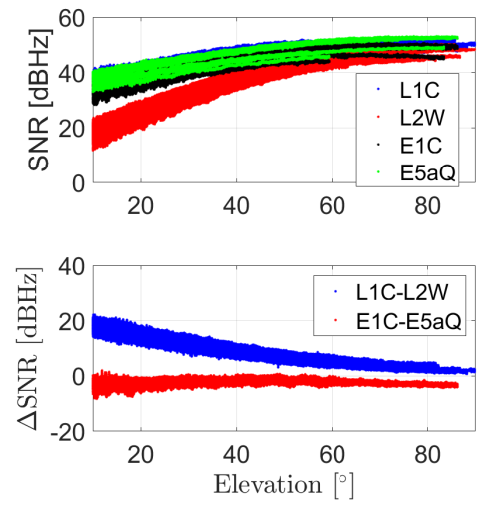

(a) Open-sky scenario

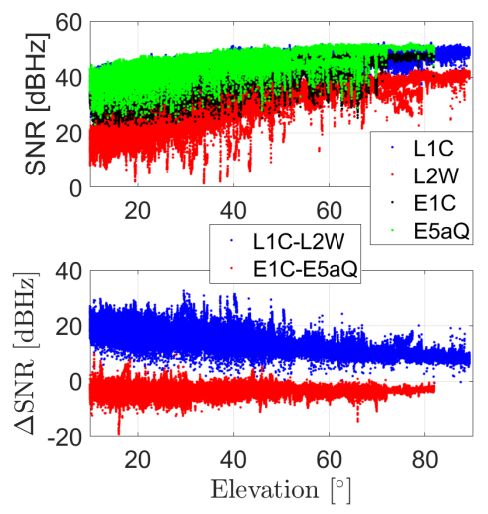

(b) Suburban scenario

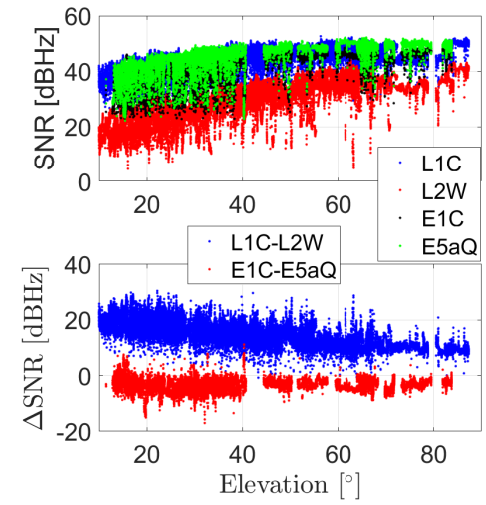

(c) Urban scenario

Fig. 4 Raw SNRs on the two frequencies and the corresponding differences with respect to the elevation angles in (a) the open-sky scenario, (b) the suburban scenario and (c) the urban scenario.

where $\log _{10}(\cdot)$ is the base 10 logarithm of the element in $(\cdot)$. Note that the smoothed observations with $\bar{S}_{k}^{s}$ $(k=u, v)$ lower than $12 \mathrm{dBHz}$, which is identified as the minimum possible signal strength in RINEX 3.03 (2015), are not used in the data analysis and processing.

Compared to the GPS L1C and Galileo E1C signals, the GPS L2W and Galileo E5aQ signals, which are used for the second frequency in this study, experience different levels of SNRs. The top panels of Figure 4 show the raw SNRs of GPS L1C, L2W, and Galileo E1C, E5aQ signals in different measurement scenarios, and the bottom panels illustrate the differences between the SNRs for the two frequencies of the GPS and the Galileo signals. Due to the different minimum received power of these signals (Zhou et al. 2019), the SNRs on L2W are generally lower than those on L1C for GPS satellites, while the SNRs on E5aQ are generally higher than those on E1C for Galileo. From Figure 4 it can also be observed that the SNRs increase smoothly with the elevation angles under the open-sky scenario, while larger randomness can be observed in the suburban and urban scenarios. This suggests again that the elevation-dependent weighting model might not be enough for the complicated measurement environments experienced in the suburban and urban scenarios, and the SNR-related weighting coefficients may need to be investigated separately for different constellations and tracking channels.

It is assumed that the variance of the combined noise and multipath for the smoothed code measurements from satellite $s$ can be formulated as:

$$
\sigma_{s}^{2}=\frac{\sigma_{0}^{2}}{w^{s}}
$$

where $\sigma_{0}$ is the zenith-referenced standard deviation for the combined noise and multipath in the IF smoothed observations, and if related to the SNRs and the number of the smoothing epochs $n$, it is assumed 
at a SNR of $\infty$ and at $n=N$. The first variant of the tested weighting models at $t_{i}$, denoted as $w_{A}^{s}$, is only related to the elevation angles $\theta^{s}$ at $t_{i}$ (in degrees), and is given as (Euler and Goad 1991):

$$
w_{A}^{s}\left(\theta^{s}\left(t_{i}\right)\right)=\frac{1}{\left(1+\alpha \times \exp \left(-\frac{\theta^{s}\left(t_{i}\right)}{10^{\circ}}\right)\right)^{2}}
$$

where $\exp (\cdot)$ represents the natural exponential function. Here by setting the selected parameter $\alpha$ in Eq. 8, the impact of the elevation angles is allowed to vary in different environments according to their conditions. Note that in this study the exponential from of the elevation-dependent weighting function is used as in EUROCAE (2019). Setting the coefficient $\alpha$ to zero would disable the elevation-dependent weighting.

In addition to the elevation angles, the second variant of the weighting model, denoted as $w_{B}^{s}$, considers the elevation angles $\left(\theta^{s}\right)$, the average SNRs $\left(\bar{S}_{k}^{s}\right)$ within the smoothing time, and the number of the smoothing epochs $n$. Assuming that $w_{A}^{s}\left(\theta^{s}\left(t_{i-n+1}\right)\right) \approx \cdots \approx w_{A}^{s}\left(\theta^{s}\left(t_{i}\right)\right)$, and the signal standard deviations $\sigma_{0, k, \text { raw }}$ are equal on both frequencies, the term $\sigma_{s}^{2}$ can be formulated as:

$$
\sigma_{s}^{2}=\frac{\sigma_{0}^{2}}{w_{B}^{s}}
$$

with the weighting model $w_{B}^{s}$ at $t_{i}$ given as (Appendix A):

$$
w_{B}^{s}\left(\theta^{s}\left(t_{i}\right), \bar{S}_{k}^{s}\left(t_{i}\right), n\right) \approx w_{A}^{s}\left(\theta^{s}\left(t_{i}\right)\right) \times w_{B 1}^{s}(n) \times w_{B 2}^{s}\left(\bar{S}_{k}^{s}\left(t_{i}\right)\right)
$$

with

$$
\begin{aligned}
w_{B 1}^{s}(n) & =\frac{1}{1+\frac{N-n}{n}} \\
w_{B 2}^{s}\left(\bar{S}_{k}^{s}\left(t_{i}\right)\right) & =\frac{1}{1+\frac{f_{u}^{4}}{f_{u}^{4}+f_{v}^{4}} \times m_{u} \times 10^{-0.1 \times \bar{S}_{u}^{s}\left(t_{i}\right)}+\frac{f_{v}^{4}}{f_{u}^{4}+f_{v}^{4}} \times m_{v} \times 10^{-0.1 \times \bar{S}_{v}^{s}\left(t_{i}\right)}}
\end{aligned}
$$

where $f_{u}$ and $f_{v}$ are the two frequencies used to form the IF combination. As the combined noise and multipath does not behave like the Gaussian white noise, and the phase noise and multipath also play a role in the smoothed observations, a parameter $\beta$ is added before the term $(N-n) / n$ to adjust the smoothing impact in general on the weighting model. The $w_{B 1}^{s}(n)$ is re-formulated as:

$$
w_{B 1}^{s}(n)=\frac{1}{1+\beta \times \frac{N-n}{n}}
$$

By varying the parameters $\alpha, m_{k}$ and $\beta$, the proposed weighting model $w_{B}^{s}$ allows a varying impact of the elevation angles, the average SNRs and the smoothing time. The impact of these factors could also be set to zeros, when $\alpha, m_{k}$ and $\beta$ are set to zeros. In general, a high elevation angle, a large SNR, and a long smoothing time would lead to a large weight $\left(w_{B}^{s}\right)$ and thus a small variance, i.e., good precision. It can be observed that when $n=N$, the term $1+\beta \times(N-n) / n$ equals to 1 and no further de-weighting is applied with respect to the carrier-smoothing.

\subsection{Weighting coefficients}

If the weighting function and the $\sigma_{0}^{2}$ could perfectly describe the variance $\sigma_{s}^{2}$ in Eq. 7 , the combined weighted and normalised noise and multipath, denoted by $\Delta \tilde{p}_{v 0}^{s}$, is expected to follow a standard normal distribution $\mathcal{N}(0,1)$ :

$$
\Delta \tilde{p}_{v 0}^{s}=\frac{\Delta \tilde{p}^{s}}{\sigma_{s}}=\frac{\Delta \tilde{p}^{s} \times \sqrt{w^{s}}}{\sigma_{0}}=\frac{\Delta \tilde{p}_{v}^{s}}{\sigma_{0}} \sim \mathcal{N}(0,1)
$$

where $\Delta \tilde{p}_{v}^{s}$ represents the combined weighted noise and multipath. All usable satellites $s$ at time $t_{i}$, with $i$ varying from the first to the last processing epochs, can be taken into consideration. With appropriate parameters chosen for the weighting model, the empirical CDF of $\Delta \tilde{p}_{v 0}^{s}$ is expected to possibly match the formal $\mathrm{CDF}$, i.e., here the $\mathrm{CDF}$ of a standard normal distribution. 
To evaluate the suitability of the weighting model, the mean shift in $\Delta \tilde{p}_{v 0}^{s}$ between the empirical and formal CDFs, denoted here as $\Delta \bar{x}$, is proposed to be used here as:

$$
\Delta \bar{x}=\operatorname{mean}\left\{\left|\Delta x_{j}^{L}\right|_{j=1, \cdots, N_{b}-1},\left|\Delta x_{j}^{R}\right|_{j=N_{b}, \cdots, 2}\right\}
$$

with the shifts in $\Delta \tilde{p}_{v 0}^{s}$ between the left- and right-folded empirical and formal CDFs, denoted as $\Delta x_{j}^{L}$ and $\Delta x_{j}^{R}$ respectively, computed as:

$$
\begin{aligned}
& \Delta x_{j}^{L}=C_{f L}^{-1}\left(C_{e L}\left(\Delta \tilde{p}_{v 0, j}^{s}\right)\right)-\Delta \tilde{p}_{v 0, j}^{s} \\
& \Delta x_{j}^{R}=C_{f R}^{-1}\left(C_{e R}\left(\Delta \tilde{p}_{v 0, j}^{s}\right)\right)-\Delta \tilde{p}_{v 0, j}^{s}
\end{aligned}
$$

where $\Delta \tilde{p}_{v 0, j}^{s}$ denotes the $j$-th value of the $\Delta \tilde{p}_{v 0}^{s}$ used for the analysis. $C_{e L}(\cdot)$ and $C_{e R}(\cdot)$ represents the leftand right-folded empirical CDFs of $\Delta \tilde{p}_{v 0}^{s}$ evaluated at the value contained in $(\cdot)$, respectively. $C_{f L}^{-1}(\cdot)$ and $C_{f R}^{-1}(\cdot)$ is the inverse function of the left- and right-folded formal CDF of the standard normal distribution at the value in $(\cdot)$, respectively. With $N_{b}$ denoting the number of samples, $j$ varies from 1 to $N_{b}-1$ for the left-folded CDF, and downwards from $N_{b}$ to 2 for the right-folded CDF. The last and the first element is not taken into consideration for the left- and the right-folded CDF, respectively, to avoid an inverse CDF of $\infty$; the mean $(\cdot)$ computes the mean value out of all the elements contained in $(\cdot)$.

Figure 5 shows the empirical and formal CDFs using data collected from Galileo satellites in the kinematic environment, i.e., from a moving vehicle over several different time periods in the urban areas of Sydney, Australia, in April, 2018. The smoothing window $N$ is set to 900 epochs. The term $\Delta x_{j}^{L}$ in Eq. 15 can, e.g., be explained by the differences in the x-values between the blue and the red lines for a certain y-value, i.e., $C_{e L}\left(\Delta \tilde{p}_{v 0, j}^{s}\right)$. Here the shifts in the x-values are used instead of those in the y-values, so that large tails could have stronger influence on the $\Delta \bar{x}$, and an easier search of the overbounding CDFs for the weighted noise and multipath can be enabled in the next sub-section. To obtain the best match between the blue and the red lines, i.e., the minimum $\Delta \bar{x}$ (Eq. 14), the $\sigma_{0}$ is empirically searched for $w_{A}^{s}$ from $0.05 \mathrm{~m}$ to $0.5 \mathrm{~m}$ with a step of $0.01 \mathrm{~m}$, and $\alpha$ is searched from 0 to 10 with a step of 1 . As $w_{B}^{s}$ requires a 5 -dimensional search, a search algorithm with shrinking space is performed. To speed up the computational load, the coefficients of $w_{B}^{s}$ are searched with relatively large steps in the first round, and in the second round with smaller steps near the potential coefficient combinations delivering low $\Delta \bar{x}$, here tested for the 30 lowest $\Delta \bar{x}$. As $w_{B}^{s}$ provides a larger degree of freedom than $w_{A}^{s}$ during the empirical search, to enable an efficient search of the overbounding CDF in the next sub-section, the coefficient combinations delivering $\left|\tilde{p}_{v 0}^{s}\right|$ exceeding 40 and 25 are not considered for $w_{B}^{s}$ anymore in the first and the second rounds, respectively. The boundaries of the search areas for the first round, i.e., $0.5 \mathrm{~m}$ for $\sigma_{0}, 10$ for $\alpha, 60 \mathrm{dBHz}$ for $\tilde{m}_{u}$ and $\tilde{m}_{v}$, and 0.5 for $\beta$ are set based on experiences, considering also the computational efficiency. This will be discussed later with Tables 1 and 2. As shown in Figure 5, the minimum $\Delta \bar{x}$ is reduced from 0.270 applying $w_{A}^{s}$ to 0.133 applying $w_{B}^{s}$. The large tails in Figure 5a (extending to above 35 and below -20) are reduced by considering the impact of the SNRs and the smoothing time.

The parameters in the weighting models vary with the measurement environments. Table 1 lists the minimal $\Delta \bar{x}$ obtained using GPS L1C and L2W signals for different smoothing windows in the opensky scenario, the suburban scenario and the urban scenario. The corresponding weighting coefficients for computing the minimal $\Delta \bar{x}$ are also shown in the table. The outlier rate describes the percentages of the measurements screened out due to the low $\bar{S}_{k}^{s}$ below $12 \mathrm{dBHz}$ and the large residuals $\Delta \tilde{p}^{s}$ above $10 \mathrm{~m}$. $\tilde{m}_{k}$ is given in $\mathrm{dBHz}$ with $\tilde{m}_{k}=10 \times \log _{10}\left(m_{k}\right)$. Recall that $\alpha, m_{k}$ and $\beta$ reflect the influences of the elevation angles, the smoothed SNRs and the smoothing time on the weighting model, respectively. In aviation, a smoothing time of $100 \mathrm{~s}$ is used according to EUROCAE (2019). For ground-based applications, a longer smoothing time is applicable to further reduce biases caused by, e.g., large multipath. In this study, smoothing windows of $360 \mathrm{~s}, 600 \mathrm{~s}$ and $900 \mathrm{~s}$ are tested for this purpose.

Having a look at the statistics applying $w_{A}^{s}$ (Table 1) for the GPS satellites, an increasing $\sigma_{0}$ can be observed with the increasing environment complexity. In the urban scenario, the residuals become less dependent on the elevation angles compared to those in the open-sky and the suburban scenarios. While the $\Delta \bar{x}$ are the same applying $w_{A}^{s}$ and $w_{B}^{s}$ in the open-sky scenario, obvious reduction of $\Delta \bar{x}$ can be observed in the suburban and urban scenarios applying $w_{B}^{s}$, which indicate a better match between the empirical and the formal CDFs of $\Delta \tilde{p}_{v 0}^{s}$ (see Figure 5). Compared to the open-sky scenario applying $w_{B}^{s}$, where an elevation-dependent weighting model seems to be sufficient, larger impacts of the smoothing time and the SNRs need to be considered in $w_{B}^{s}$ for the suburban and urban scenarios. To be noted is that after applying 


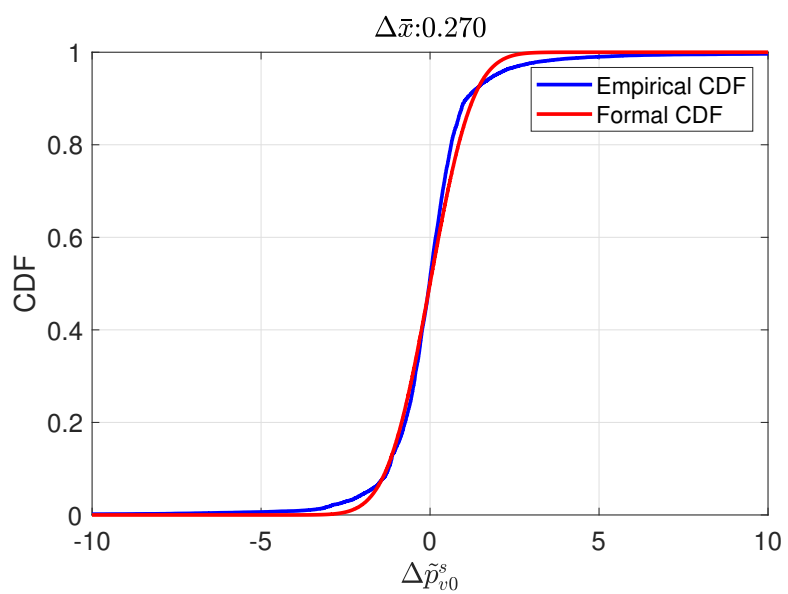

(a) $w_{A}^{s}$

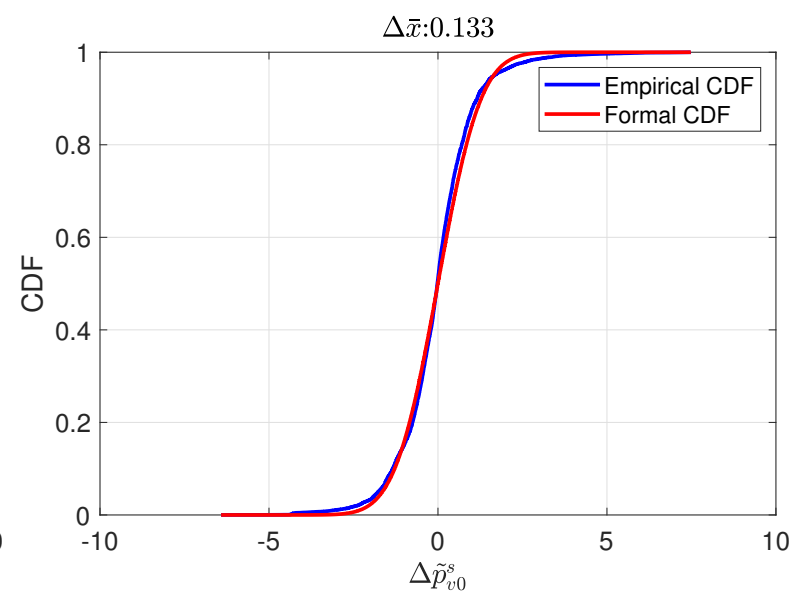

(b) $w_{B}^{s}$

Fig. 5 Formal and empirical CDFs of the $\Delta \tilde{p}_{v 0}^{s}$ (Eq. 13) applying the weighting functions (a) $w_{A}^{s}$ and (b) $w_{B}^{s}$. Data observed from all available Galileo satellites for the experiments in the urban areas of Sydney were used with a smoothing window $N$ of 900 epochs. The x-axes are scaled to -10 and 10 for the purpose of visualisation

Table 1 Parameters and statistics applying the weighting functions $w_{A}^{s}$ and $w_{B}^{s}$ for GPS satellites under different measurement scenarios. Note that $\tilde{m}_{u}$ and $\tilde{m}_{v}$ are given in $\mathrm{dBHz}$ with $\tilde{m}_{u}=10 \times \log _{10}\left(m_{u}\right)$ and $\tilde{m}_{v}=10 \times \log _{10}\left(m_{v}\right)$

\begin{tabular}{c|c|c|c|c|c|c|c|c|c|c|c}
\hline Scenario & $N$ & Outlier & \multicolumn{3}{|c|}{$w_{A}^{s}$} & \multicolumn{8}{|c}{$w_{B}^{s}$} \\
\hline & & & $\sigma_{0}[\mathrm{~m}]$ & $\alpha$ & $\Delta \bar{x}$ & $\sigma_{0}[\mathrm{~m}]$ & $\alpha$ & $\begin{array}{c}\tilde{m}_{u} \\
{[\mathrm{dBHz}]}\end{array}$ & $\begin{array}{c}\tilde{m}_{v} \\
{[\mathrm{dBHz}]}\end{array}$ & $\beta$ & $\Delta \bar{x}$ \\
& & & & & & & & & & \\
\hline Open-sky & 360 & $0 \%$ & 0.22 & 5 & 0.052 & 0.22 & 5 & $-\infty$ & $-\infty$ & 0.0 & 0.052 \\
scenario & 600 & $0 \%$ & 0.18 & 6 & 0.062 & 0.18 & 6 & $-\infty$ & $-\infty$ & 0.0 & 0.062 \\
& 900 & $0 \%$ & 0.17 & 4 & 0.058 & 0.17 & 4 & $-\infty$ & $-\infty$ & 0.0 & 0.058 \\
\hline Suburban & 360 & $2.5 \%$ & 0.24 & 4 & 0.161 & 0.20 & 2 & 36 & 18 & 0.05 & 0.144 \\
scenario & 600 & $2.5 \%$ & 0.24 & 3 & 0.169 & 0.18 & 2 & 36 & 10 & 0.05 & 0.144 \\
& 900 & $2.5 \%$ & 0.22 & 4 & 0.173 & 0.16 & 2 & 36 & 14 & 0.05 & 0.141 \\
\hline Urban & 360 & $2.8 \%$ & 0.36 & 3 & 0.349 & 0.18 & 0 & 24 & 28 & 0.25 & 0.298 \\
scenario & 600 & $2.9 \%$ & 0.36 & 3 & 0.359 & 0.17 & 0 & 26 & 26 & 0.2 & 0.305 \\
& 900 & $3.1 \%$ & 0.38 & 2 & 0.365 & 0.15 & 0 & 20 & 28 & 0.15 & 0.313 \\
\hline
\end{tabular}

$w_{B}^{s}$, the large $\sigma_{0}$ (in the zenith direction at a SNR of $\infty$ ), e.g., in the urban scenario is strongly reduced. The large multipath in such environment is better described by the more comprehensive weighting model $w_{B}^{s}$, and is less absorbed by the $\sigma_{0}$. From Table 1 it can also be observed that the boundary values set for coefficient search are not exceeded.

The situation for the Galileo signals is a bit different. Table 2 shows the statistics applying $w_{A}^{s}$ and $w_{B}^{s}$ using the Galileo E1C and E5aQ signals. It can be observed that the $w_{A}^{s}$, depending only on the elevation angles, becomes more effective in the urban scenario compared to the case of GPS. This leads to similar $\sigma_{0}$ under different environments, and larger $\alpha$ in the urban scenario, which indicates large residuals at low elevation angles. Considering the fact that $\alpha$ has not reached the limit of 10 in case of a smoothing time of $360 \mathrm{~s}$ in the urban scenario, and the $\alpha$ for other scenarios and for the GPS satellites does not differ much for different smoothing windows, a larger $\alpha$ above 10 is not further attempted in this study. Similar as for the GPS satellites, applying $w_{B}^{s}$ for Galileo satellites can also lead to reduction in $\Delta \bar{x}$, which is a result of increasing the impact of the SNRs and the smoothing time. Note that $\Delta \bar{x}$ is insensitive to the smoothing time in the open-sky scenario, since only a small proportion of the data occupies small $n$ there. As such, search with a higher $\beta$ above 0.5 is not further attempted here.

In general, applying $w_{B}^{s}$ is able to better match the formal and the empirical CDFs of $\Delta \tilde{p}_{v 0}^{s}$, and the improvement increases with the increasing measurement complexity. Compared to the open-sky scenario 
Table 2 Parameters and statistics applying the weighting functions $w_{A}^{s}$ and $w_{B}^{s}$ for Galileo satellites under different measurement scenarios. Note that $\tilde{m}_{u}$ and $\tilde{m}_{v}$ are given in $\mathrm{dBHz}$ with $\tilde{m}_{u}=10 \times \log _{10}\left(m_{u}\right)$ and $\tilde{m}_{v}=10 \times \log _{10}\left(m_{v}\right)$

\begin{tabular}{c|c|c|c|c|c|c|c|c|c|c|c}
\hline Scenario & $N$ & Outlier & \multicolumn{3}{|c|}{$w_{A}^{s}$} & \multicolumn{1}{c}{$w_{B}^{s}$} \\
\hline & & & $\sigma_{0}[\mathrm{~m}]$ & $\alpha$ & $\Delta \bar{x}$ & $\sigma_{0}[\mathrm{~m}]$ & $\alpha$ & $\begin{array}{c}\tilde{m}_{u} \\
{[\mathrm{dBHz}]}\end{array}$ & $\begin{array}{c}\tilde{m}_{v} \\
{[\mathrm{dBHz}]}\end{array}$ & $\beta$ & $\Delta \bar{x}$ \\
& & & & & & & & & & \\
\hline Open-sky & 360 & $0 \%$ & 0.18 & 5 & 0.032 & 0.13 & 1 & 32 & 54 & 0.30 & 0.028 \\
scenario & 600 & $0 \%$ & 0.15 & 5 & 0.038 & 0.14 & 3 & $-\infty$ & 46 & 0.50 & 0.036 \\
& 900 & $0 \%$ & 0.12 & 6 & 0.050 & 0.10 & 1 & 34 & 52 & 0.50 & 0.043 \\
\hline Suburban & 360 & $0.003 \%$ & 0.21 & 2 & 0.189 & 0.19 & 0 & 22 & 36 & 0.05 & 0.169 \\
scenario & 600 & $0.003 \%$ & 0.18 & 3 & 0.192 & 0.16 & 0 & $-\infty$ & 38 & 0.05 & 0.166 \\
& 900 & $0.003 \%$ & 0.16 & 4 & 0.197 & 0.13 & 1 & 20 & 36 & 0.05 & 0.163 \\
\hline Urban & 360 & $0.4 \%$ & 0.20 & 9 & 0.262 & 0.04 & 0 & 56 & 58 & 0.05 & 0.158 \\
scenario & 600 & $0.4 \%$ & 0.19 & 10 & 0.264 & 0.06 & 0 & 50 & 56 & 0.05 & 0.152 \\
& 900 & $0.4 \%$ & 0.17 & 10 & 0.270 & 0.04 & 0 & 54 & 54 & 0.05 & 0.133 \\
\hline
\end{tabular}

where similar $\Delta \bar{x}$ are delivered by both weighting functions, considering the impacts of both the SNRs and the smoothing time shows to be important for the processing in the suburban and urban scenarios.

\subsection{Overbounding $\mathrm{CDF}$}

In the positioning process and integrity monitoring, the measurement errors are expected to follow a zeromean Gaussian normal distribution. The distribution of the empirical errors, however, could be non-zeromean, unsymmetric and multimodal. To monitor the integrity, an overbounding normal distribution that conservatively covers all the errors is thus required. In this section, the focus is on the overbounding CDFs for the combined weighted noise and multipath $\Delta \tilde{p}_{v}^{s}$ (Eq. 13). The scenario varies for different smoothing windows from 360 to 900 epochs, and for different measurement environments, i.e., from the open-sky to the urban scenarios.

As introduced in Rife et al. (2004, 2006), the paired overbounding CDF contains the empirical CDF in envelope-like left and right CDFs, which share the same overbounding standard deviation $\sigma_{o b}$ and have opposite overbounding mean values $m_{o b}$. Different from the DeCleene's overbounding method (DeCleene 2000) that uses a single CDF as the overbounding function, the paired overbounding function does not restrict the shape of the empirical distribution, and guarantees the observation-to-position conservatism of the overbounding characteristics. As a generalisation of the paired overbounding function, the excess-mass CDF (EMC) (Rife et al. 2004) allows a total mass of the overbounding functions larger than 1 and thus releases the strict requirements in the paired overbounding. The left and right EMCs, denoted as $G_{L}$ and $G_{R}$, can be formulated as:

$$
\begin{aligned}
& G_{L}(x)=\int_{-\infty}^{x}(1+\epsilon) \mathcal{N}\left(-m_{o b}, \sigma_{o b}\right) d x \geq G_{a}(x) \\
& G_{R}(x)=\int_{-\infty}^{x}(1+\epsilon) \mathcal{N}\left(m_{o b}, \sigma_{o b}\right) d x-\epsilon \leq G_{a}(x)
\end{aligned}
$$

where the $\mathcal{N}\left(m_{o b}, \sigma_{o b}\right)$ stands for the normal distribution with the mean value of $m_{o b}$ and the standard deviation of $\sigma_{o b}$. The term $\epsilon$ represents the excess mass, which will be considered later by computing the protection levels.

Examples are given in Figure 6 for $\Delta \tilde{p}_{v}^{s}$ of Galileo satellites in the urban scenario with a smoothing window of 360 epochs. The weighting functions $w_{A}^{s}$ and $w_{B}^{s}$ with the parameters given in Table 2 were applied to form the $\Delta \tilde{p}_{v}^{s}$. The overbounding mean value $m_{o b}$ and the overbounding standard deviation $\sigma_{o b}$ were searched in loops. The $m_{o b}$ was incremented from 0 to $3 \mathrm{~m}$ with a step of $0.01 \mathrm{~m}$, and for each $m_{o b}$, the $\sigma_{o b}$ was incremented from 0 to $3 \mathrm{~m}$ with a step of $0.01 \mathrm{~m}$. The loop stops when the criteria in Eqs. 17 and 18 are fulfilled. To increase the computational efficiency, the excess mass is set in this contribution to 0.05 and not searched for a range of values. As shown in Figure 6, the empirical CDFs (the blue lines) are 


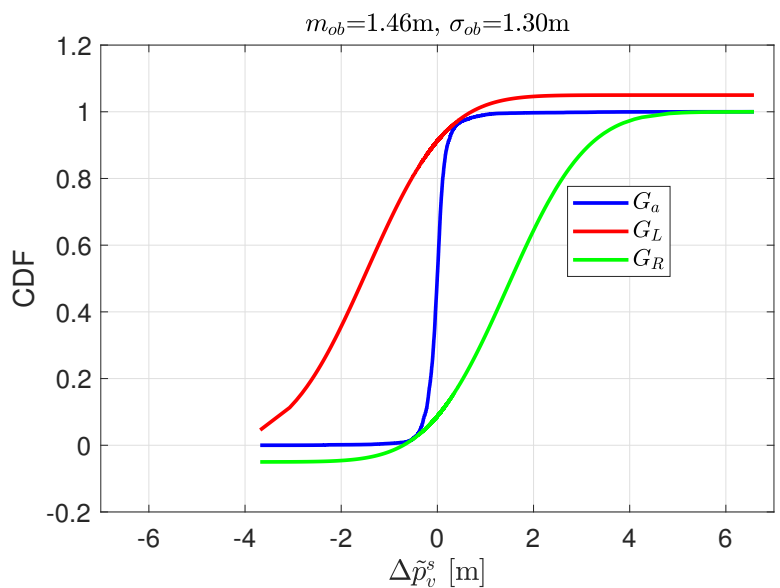

(a) $w_{A}^{s}$

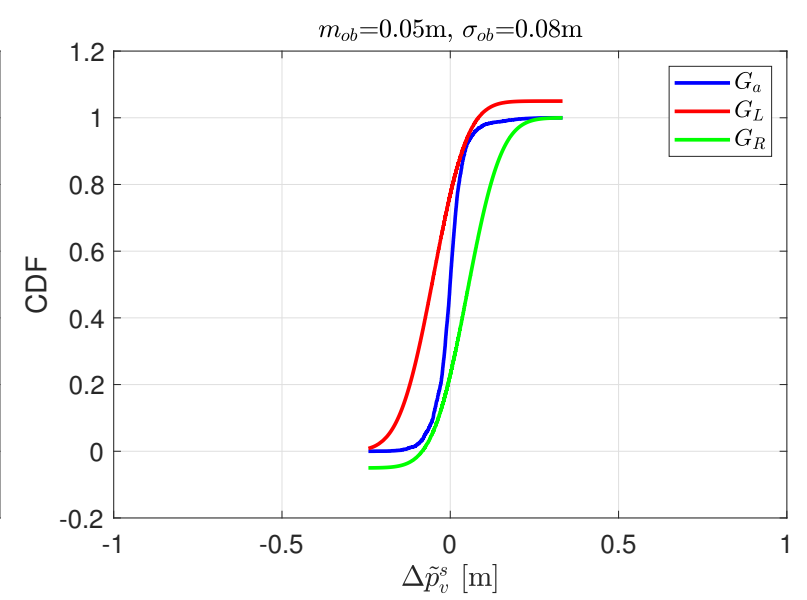

(b) $w_{B}^{s}$

Fig. 6 EMC overbounding functions for $\Delta \tilde{p}_{v}^{s}$ of Galileo satellites applying the weighting functions: (a) $w_{A}^{s}$ and (b) $w_{B}^{s}$ in the urban scenario with a smoothing window $N$ of 360 epochs. Note that the x-scales of the sub-figures are different

Table 3 The overbounding mean values $m_{o b}$ and standard deviations $\sigma_{o b}$ for $\Delta \tilde{p}_{v}^{s}$ of GPS satellites in different measurement environments

\begin{tabular}{c|c|c|c|c|c|c}
\hline Scenario & \multicolumn{3}{c|}{$m_{o b}[\mathrm{~m}]$} & \multicolumn{3}{c}{$\sigma_{o b}[\mathrm{~m}]$} \\
\hline & $N=360$ & $N=600$ & $N=900$ & $N=360$ & $N=600$ & $N=900$ \\
\hline \multicolumn{7}{c|}{$w_{A}^{s}$} \\
\hline Open-sky scenario & 0.08 & 0.07 & 0.12 & 0.33 & 0.27 & 0.30 \\
\hline Suburban scenario & 1.29 & 1.44 & 1.37 & 1.28 & 1.34 & 1.27 \\
\hline Urban scenario & 2.11 & 2.11 & 2.12 & 2.06 & 2.06 & 2.09 \\
\hline \multicolumn{7}{c}{$w_{B}^{s}$} \\
\hline Open-sky scenario & 0.08 & 0.07 & 0.12 & 0.33 & 0.27 & 0.30 \\
\hline Suburban scenario & 0.29 & 0.24 & 0.21 & 0.49 & 0.42 & 0.37 \\
\hline Urban scenario & 0.82 & 0.74 & 0.69 & 0.82 & 0.75 & 0.69 \\
\hline
\end{tabular}

enclosed within the left (red) and right (green) overbounding CDFs. Applying the weighting function $w_{B}^{s}$ significantly reduces the $m_{o b}$ and $\sigma_{o b}$ from metres to below $1 \mathrm{dm}$ due to the de-weighting of the large tails.

The overbounding mean values and standard deviations are given in Table 3 for the GPS satellites and in Table 4 for the Galileo satellites. A significant reduction can be observed in both the $m_{o b}$ and $\sigma_{o b}$ applying $w_{B}^{s}$ for the suburban and urban scenarios, while the values in the open-sky scenario applying $w_{B}^{s}$ do not change for GPS and reduced by centimetres for Galileo. As the majority of the observations are from GPS, this suggests that an elevation-dependent weighting model could already be enough for the open-sky scenario, while considering the impacts of the SNRs and the smoothing time is important for de-weighting the large tails and reducing the parameters in EMCs for applications in the suburban and urban scenarios. It is noted that the analysis is performed using $1 \mathrm{~Hz}$ data in this study. For data with higher sampling rate, the same length of the smoothing window in time would imply more smoothing epochs, and thus smaller smoothed noise and multipath. The overbounding standard deviations and mean values generated for $1 \mathrm{~Hz}$ data are thus expected to bound the better smoothed measurement residuals when using the proposed weighting model.

\section{Observation model}

For the SBAS DFMC positioning service using GPS and Galileo, the expectation of the observed-minuscomputed (O-C) terms of the carrier-smoothed IF code measurements $y$ can be formulated with the following 
Table 4 The overbounding mean values $m_{o b}$ and standard deviations $\sigma_{o b}$ for $\Delta \tilde{p}_{v}^{s}$ of Galileo satellites in different measurement environments

\begin{tabular}{c|c|c|c|c|c|c}
\hline Scenario & \multicolumn{3}{c|}{$m_{o b}[\mathrm{~m}]$} & \multicolumn{3}{c}{$\sigma_{o b}[\mathrm{~m}]$} \\
\hline & $N=360$ & $N=600$ & $N=900$ & $N=360$ & $N=600$ & $N=900$ \\
\hline \multicolumn{7}{c|}{$w_{A}^{s}$} \\
\hline Open-sky scenario & 0.03 & 0.05 & 0.07 & 0.23 & 0.22 & 0.20 \\
\hline Suburban scenario & 1.51 & 1.55 & 1.56 & 1.37 & 1.35 & 1.33 \\
\hline Urban scenario & 1.46 & 1.43 & 1.43 & 1.30 & 1.26 & 1.26 \\
\hline \multicolumn{7}{c|}{$w_{B}^{s}$} \\
\hline Open-sky scenario & 0.02 & 0.01 & 0.02 & 0.17 & 0.17 & 0.13 \\
\hline Suburban scenario & 0.31 & 0.23 & 0.18 & 0.48 & 0.38 & 0.31 \\
\hline Urban scenario & 0.05 & 0.06 & 0.05 & 0.08 & 0.10 & 0.08 \\
\hline
\end{tabular}

observation equations:

$$
\mathrm{E}(y)=\underbrace{\left[G, e_{G}, e_{E}\right]}_{A} \cdot\left[\begin{array}{c}
\Delta r \\
t_{G} \\
t_{E}
\end{array}\right]
$$

where the design matrix $A$ is constructed with the geometry matrix $G$, and the vectors $e_{G}$ and $e_{E}$. $G=$ $\left[u^{1}, \cdots, u^{l}\right]^{T}$ contains the 3 -dimensional north-east-up satellite-to-receiver unit vectors $u^{s}$ for $s=1, \cdots, l$, with $l$ denoting the number of satellites. The vectors $e_{G}$ and $e_{E}$ contains the element 1 when the corresponding observation is from a GPS and a Galileo satellite, respectively. Other elements in $e_{G}$ and $e_{E}$ are set to zeros. The terms $\Delta r, t_{G}$ and $t_{E}$ are the 3 -dimensional receiver positioning increments, and the GPS and the Galileo receiver clock offsets. $\mathrm{E}(\cdot)$ is the expectation operator. With the help of the broadcast SBAS messages, the orbital and satellite clock corrections are applied to the orbits and clocks computed using the broadcast ephemeris. The approximate receiver coordinates, the corrected satellite orbits and clocks, the relativistic effects, the corrections of the phase centre offsets (PCOs) and the phase centre variations $(\mathrm{PCVs})$ are then used to compute the modelled ranges. The group delays are to be considered depending on the system and the frequencies used. The modelled ranges are next subtracted from the smoothed IF code observations $\tilde{p}_{I F}^{s}$ to form the O-C term $y$ in Eq. 19.

The variance-covariance matrix of the $\mathrm{O}-\mathrm{C}$ terms is assumed to be a diagonal matrix, such that:

$$
Q_{y}=\operatorname{diag}\left(\sigma_{a, 1}^{2}, \cdots, \sigma_{a, l}^{2}\right)
$$

where $\operatorname{diag}(\cdot)$ forms a diagonal matrix with the diagonal elements contained in $(\cdot)$. The variance element $\sigma_{a, s}^{2}$ for satellite $s$ consists of four parts as follows:

$$
\sigma_{a, s}^{2}=\frac{\left(\sigma_{o b}^{s}\right)^{2}}{w^{s}}+\left(\sigma_{\tau}^{s}\right)^{2}+\left(\sigma_{\iota}^{s}\right)^{2}+\left(\sigma_{D F C}^{s}\right)^{2}
$$

where $\sigma_{o b}^{s}$ is the overbounding standard deviation of the corresponding constellation that includes satellite $s . \sigma_{\tau}^{s}$ is the standard deviation of the tropospheric residuals that are not modelled and corrected in the O-C terms, which can be approximated for satellite $s$ as (EUROCAE 2019):

$$
\sigma_{\tau}^{s}=0.12 \times \frac{1.001}{\sqrt{0.002001+\sin ^{2}\left(\theta^{s} \times \frac{\pi}{180^{\circ}}\right)}}
$$

The term $\sigma_{\iota}^{s}$ denotes the standard deviation of the remaining ionospheric residuals after removing the first-order ionospheric delays through the IF linear combination. Its form follows as (EUROCAE 2019):

$$
\sigma_{\iota}^{s}=\frac{40}{261+\left(\theta^{s}\right)^{2}}+0.018
$$

The last term $\sigma_{D F C}^{s}$ stands for the standard deviation of the SBAS orbital and clock corrections mapped into the observation direction. It is calculated as described in EUROCAE (2019) based on the dual-frequency range error (DFRE) indicator broadcast by the SBAS satellite, the relative geometry between the user and 
the satellite, the variance-covariance matrix of the SBAS corrections and the corresponding scale factor, as well as the degradation parameters of the SBAS corrections with time.

Based on the observation equation (Eq. 19) and the variance-covariance matrix of the observations (Eq. 20), the rover positioning increments and the receiver clock errors can be computed with the leastsquares adjustment as:

$$
\Delta \hat{x}=\left[\Delta \hat{r}, \hat{t}_{G}, \hat{t}_{E}\right]^{T}=\underbrace{\left(A^{T} Q_{y}^{-1} A\right)^{-1} A^{T} Q_{y}^{-1}}_{S} y
$$

with its variance-covariance matrix $Q_{\hat{x}}$ expressed as:

$$
Q_{\hat{x}}=\left(A^{T} Q_{y}^{-1} A\right)^{-1}
$$

In this study, as we focus on land applications, we evaluate the horizontal positioning errors (HPEs) in the direction along the semi-major axis of the horizontal error ellipse $Q_{\hat{x}_{H}}$ following EUROCAE (2019), which corresponds to the north and east variance-covariance components in $Q_{\hat{x}}$. Using the first eigenvector of $Q_{\hat{x}_{H}}$, denoted as $E_{H}$, the horizontal positioning increment $\Delta \hat{x}_{H}$ can be computed as follows:

$$
\Delta \hat{x}_{H}=\left[E_{H}^{T}, 0,0,0\right] \cdot \Delta \hat{x}
$$

\section{Protection level}

In the ground-based applications, IM is important to ensure that the positioning errors are bounded by certain protection levels with a pre-defined PHMI. Following similar procedure of the SBAS DFMC positioning given in EUROCAE (2019), before computing the protection levels, the FDE procedure is performed using the $\chi^{2}$ statistic:

$$
\chi^{2}=y^{T} \cdot\left(Q_{y}^{-1}-Q_{y}^{-1} \cdot A \cdot Q_{\hat{x}} \cdot A^{T} \cdot Q_{y}^{-1}\right) \cdot y
$$

The $\chi^{2}$ statistic is then compared with the test threshold $T_{\chi^{2}}$, expressed as:

$$
T_{\chi^{2}}=Q_{\chi_{f}^{2}}^{-1}\left(1-P_{F A}\right)
$$

where $P_{F A}$ is the probability of false alert, and $Q_{\chi_{f}^{2}}^{-1}$ is the inverse CDF of the $\chi^{2}$-distribution with the degrees of freedom $f$. In case that the test statistic value $\chi^{2}$ (Eq. 27) is larger than the test threshold $T_{\chi^{2}}$, the observation corresponding to the largest value of $\hat{e}_{i} / \sigma_{\hat{e}_{i}}(i=1, \cdots, l)$ is excluded and the computation is repeated with the remaining observations until the test passes. $\sigma_{\hat{e}_{i}}$ is the standard deviation of the residuals derived from the variance-covariance matrix of the residuals $Q_{\hat{e}}$ :

$$
Q_{\hat{e}}=Q_{y}-A \cdot Q_{\hat{x}} \cdot A^{T}
$$

In correspondence with the outlier detection criterion set during signal analysis in Section 2, any observations with residuals larger than $10 \mathrm{~m}$ are assumed to experience very large multipath or NLOS errors. They are excluded before the FDE procedure.

After the FDE procedure, the protection levels are computed in the direction along the semi-major axis of the horizontal error ellipse as follows:

$$
P L_{H}=K_{H} \cdot \sqrt{E_{H}^{T} \cdot Q_{\hat{x}_{H}} \cdot E_{H}}+\left|E_{H}^{T} \cdot S_{H}\right| \cdot M_{o b}
$$

where

$$
\begin{aligned}
& M_{o b}=\left[M_{o b}^{1}, \cdots, M_{o b}^{l}\right]^{T} \\
& M_{o b}^{s}=\frac{m_{o b}^{s}}{\sqrt{w^{s}}}
\end{aligned}
$$

and $m_{o b}^{s}$ is the overbounding mean value of the corresponding constellation that includes satellite $s . S_{H}$ is constructed by the first two rows of the projector matrix $S$ (Eq. 24) that performs the transformation from the observation-domain to the position-domain. $K_{H}$ can be computed based on the horizontal PHMI $\left(\mathrm{PHMI}_{\mathrm{HOR}}\right)$ and the excess mass $\epsilon$ of the corresponding scenario (Rife et al. 2004):

$$
K_{H}=Q^{-1}\left(\frac{\mathrm{PHMI}_{\mathrm{HOR}}}{2 \times(1+\epsilon)^{n}}\right)
$$

where $Q^{-1}(\cdot)$ is the inverse function of the right-folded CDF of a standard normal distribution. Note that $\mathrm{PHMI}_{\mathrm{HOR}}$ in road transport is application-dependent. 


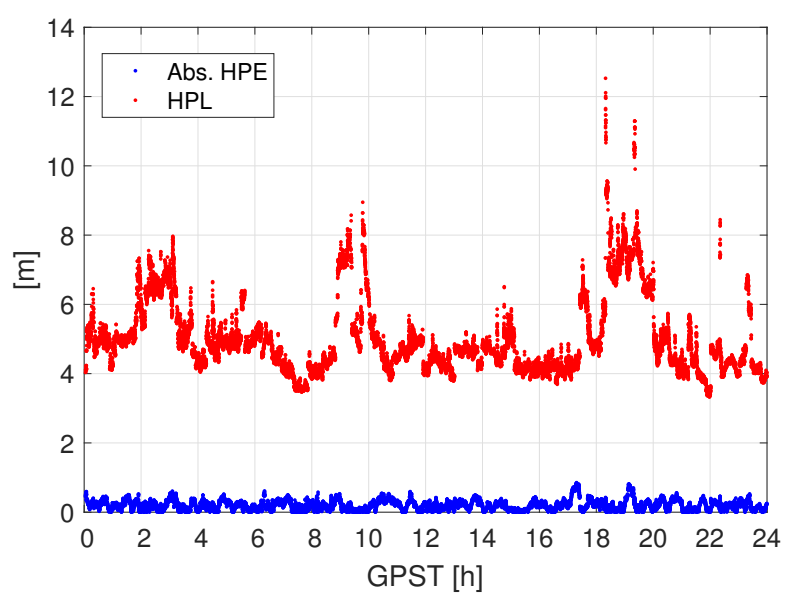

(a) HPEs and HPLs

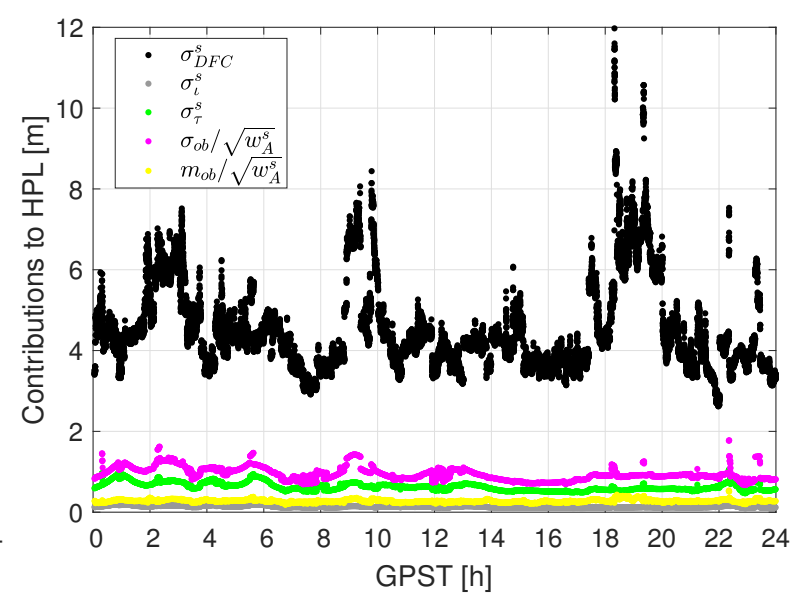

(b) Contributions to HPLs

Fig. 7 (a) The SBAS DFMC HPEs and HPLs and (b) the contributions of different noise and errors to the HPLs under the open-sky scenario. The weighting function $w_{A}^{s}$ was applied with the smoothing window $N=900$

\section{Test results}

In this section, the SBAS DFMC HPEs and HPLs are computed using $1 \mathrm{~Hz}$ GPS and Galileo data collected in different measurement environments. Recall that the weighting coefficients are obtained from Tables 1 and 2 , and the overbounding standard deviations and mean values were obtained from Tables 3 and 4 . As an example, Figure 7a shows the HPEs (blue dots) and HPLs (red dots) in the open-sky scenario. The data was collected from the static station UWA0 located in the University of Western Australia on September 14, 2018. The smoothing window is set to be 900 epochs, and the $\mathrm{PHMI}_{\mathrm{HOR}}$ is set to $10^{-5}$ (El-Mowafy and Kubo 2018). The weighting model $w_{A}^{s}$ was used for the plots with the corresponding parameters given in Tables 1 and 3. The data collected during the period 00:03:00 to 23:59:59 in GPS time (GPST) on September 14, 2018 was processed.

As shown in Figure 7a, under the open-sky scenario for the smoothing window of 900 epochs, the mean absolute HPEs amounts to about $0.2 \mathrm{~m}$. The HPLs are mostly within $8 \mathrm{~m}$, and bound the absolute HPEs during the entire processing time period. Having a look at the contributions of different error sources on the HPLs in Figure 7b, it can be observed that the $\sigma_{D F C}^{s}$, i.e., the standard deviations of the SBAS orbital and clock corrections in the signal direction plays the dominant role affecting the HPL values in the open-sky scenario. Note that the contributions of different error sources are calculated using Eqs. 30 considering only the variances or the biases listed in the legend.

Applying the weighting function $w_{B}^{s}$ for the open-sky scenario, as shown in the top panels of Figure 8a and b, no significant differences can be observed in the HPLs. For the suburban and urban scenarios, data collected from a moving vehicle in Wollongong on June 1, 2018, and in Sydney on April 19, 2018 are used for plots in the middle and bottom panels, respectively. It can be observed that the HPLs increase with the complexity of the measurement environments. This is caused by the increased multipath effects, the increased overbounding standard deviation and mean values (mapped into the slant direction), and the decreased satellite visibility as shown in Figure 8c. The average satellite number used for the processing is reduced from about 13.5 in the open-sky scenario to about 9.9 in the urban scenario. Compared to the case applying $w_{A}^{s}$ (blue dots in Figure 8a), the mean HPLs are reduced by about $40 \%$ and $14 \%$ applying $w_{B}^{s}$ (green dots) in the suburban and urban scenarios, respectively. The reduction is also clearly visible in the histograms shown in Figure 8b.

The mean absolute HPEs and the mean HPLs are given in Table 5 for different measurement environments applying the weighting functions $w_{A}^{s}$ and $w_{B}^{s}$. Note that time epochs with the number of GPS satellites lower than 4 or with the number of all satellites (from both GPS and Galileo) lower than 5 were not considered in the data processing. While similar mean HPLs are observed for the open-sky scenario applying both weighting functions, the mean HPLs are significantly reduced when applying $w_{B}^{s}$ in the suburban and urban scenarios. Among them, as shown in Table 5, the reduction of the mean HPLs amounts to about $35-40 \%$ in the suburban scenario. In the urban scenario, the mean absolute HPEs are also ob- 

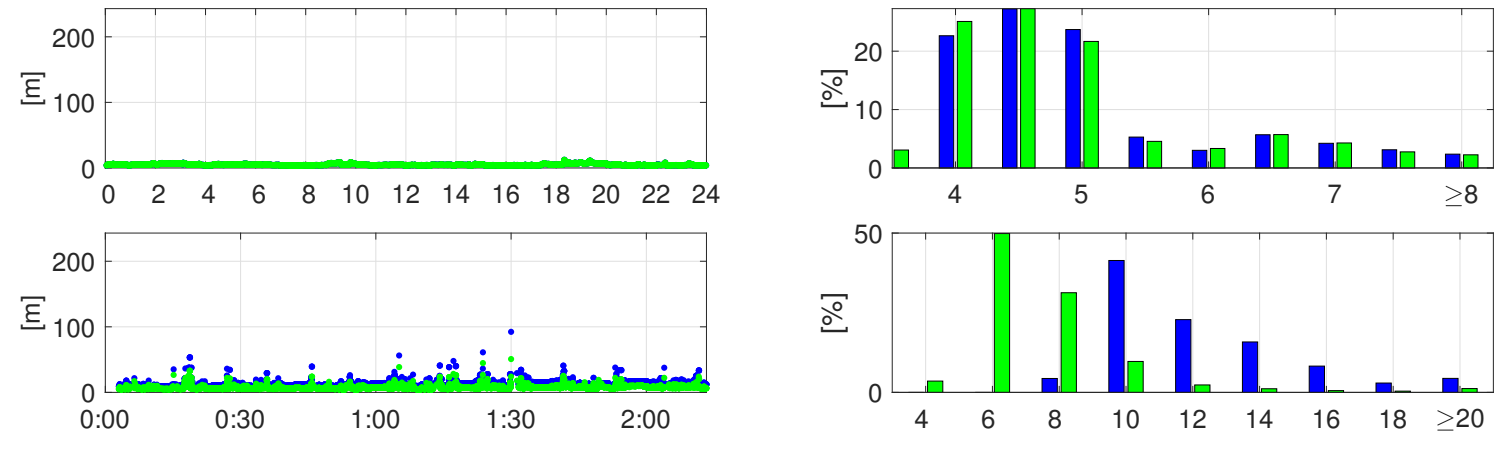

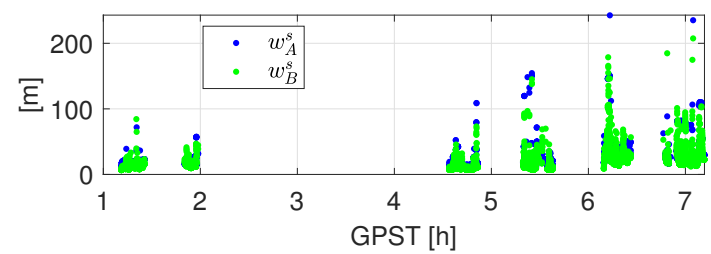

(a) HPL

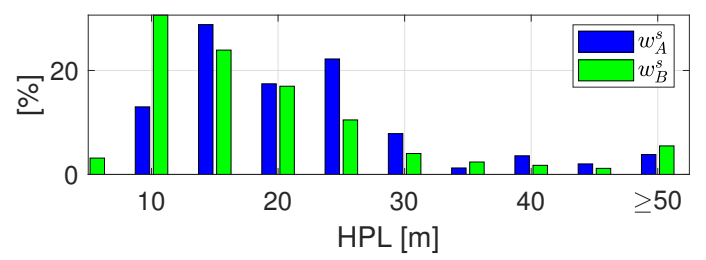

(b) HPL histogram
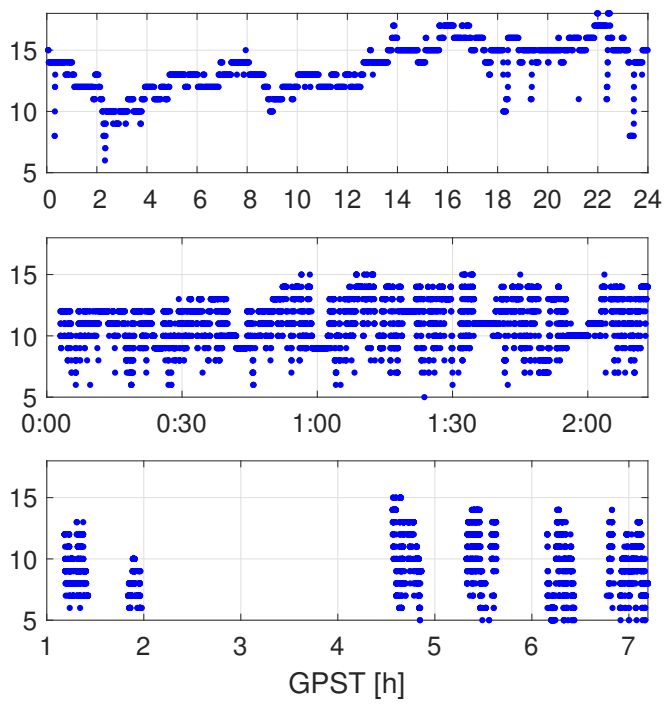

(c) Satellite No.

Fig. 8 (a) The HPLs, (b) the histograms for the HPLs and (c) the number of satellites used for the processing, in the open-sky scenario (top), in the suburban scenario (middle), and in the urban scenario (bottom). The smoothing window is 900 epochs

served to be reduced by about $10 \%$. It is remarked that, the overall much higher HPLs than the HPEs are partially caused by the bounding variances of the satellite orbit and clock errors, which are computed based on the integrity information broadcast in the SBAS message according to the ED-259 standards for aviation (EUROCAE 2019). Before releasing the ED-259 standards, a mean HPL above $5 \mathrm{~m}$, for example, was also calculated in Barrios et al. (2018) for static station in the open-sky scenario. The increasing HPLs in the suburban and urban scenarios are mainly caused by the poorer satellite visibility and the larger overbounding variances (Eq. 21) and mean values (Eq. 32) for the noise and multipath in the slant direction.

The integrity performance applying the two weighting models is evaluated as shown in Figure 9 in form of the Stanford ESA integrity diagram (Tossaint et al. 2006) assuming a horizontal alert limit (HAL) of $10 \mathrm{~m}$ and with the PHMI $\mathrm{HOR}$ equal to $10^{-5}$ as an example. The smoothing window is set to 900 epochs. The statistics are given using time epochs that have valid positioning results and HPLs, applying both 
Table 5 Mean absolute HPEs and the mean HPLs under different measurement scenarios. The values applying $w_{A}^{s}$ and $w_{B}^{s}$, and the percentage of improvement applying $w_{B}^{s}$ compared to the case applying $w_{A}^{s}$, are separated by '/', respectively

\begin{tabular}{|c|c|c|c|c|}
\hline \multirow[t]{2}{*}{$N$} & \multirow{2}{*}{$\begin{array}{c}\text { Mean abs. HPE }[\mathrm{m}] \\
\& \text { improvement }\end{array}$} & \multicolumn{3}{|c|}{ Mean HPL [m] \& improvement } \\
\hline & & $\mathrm{PHMI}_{\mathrm{HOR}}=10^{-5}$ & $\mathrm{PHMI}_{\mathrm{HOR}}=10^{-6}$ & $\mathrm{PHMI}_{\mathrm{HOR}}=10^{-7}$ \\
\hline \multicolumn{5}{|c|}{ Open-sky scenario } \\
\hline 360 & $0.22 / 0.22 / 0.1 \%$ & $4.93 / 4.94 /-0.1 \%$ & $5.42 / 5.42 /-0.1 \%$ & $5.86 / 5.87 /-0.1 \%$ \\
\hline 600 & $0.21 / 0.21 / 0.0 \%$ & $4.90 / 4.86 / 0.7 \%$ & $5.37 / 5.34 / 0.7 \%$ & $5.82 / 5.78 / 0.6 \%$ \\
\hline 900 & $0.20 / 0.20 /-0.0 \%$ & $4.98 / 4.94 / 0.9 \%$ & $5.46 / 5.42 / 0.8 \%$ & $5.90 / 5.86 / 0.7 \%$ \\
\hline \multicolumn{5}{|c|}{ Suburban scenario } \\
\hline 360 & $0.38 / 0.37 / 1.7 \%$ & $11.9 / 7.6 / 36.1 \%$ & $12.8 / 8.3 / 35.1 \%$ & $13.6 / 8.9 / 34.3 \%$ \\
\hline 600 & $0.38 / 0.37 / 1.0 \%$ & $12.3 / 7.4 / 39.7 \%$ & $13.2 / 8.1 / 38.6 \%$ & $14.0 / 8.7 / 37.7 \%$ \\
\hline 900 & $0.37 / 0.37 / 0.3 \%$ & $12.3 / 7.4 / 39.9 \%$ & $13.2 / 8.1 / 38.7 \%$ & $13.9 / 8.7 / 37.8 \%$ \\
\hline \multicolumn{5}{|c|}{ Urban scenario } \\
\hline 360 & $1.45 / 1.30 / 10.0 \%$ & $23.8 / 20.3 / 14.9 \%$ & $25.5 / 21.9 / 14.4 \%$ & $27.1 / 23.3 / 14.0 \%$ \\
\hline 600 & $1.45 / 1.33 / 8.7 \%$ & $23.6 / 19.8 / 16.3 \%$ & $25.3 / 21.3 / 15.8 \%$ & $26.9 / 22.7 / 15.5 \%$ \\
\hline 900 & $1.46 / 1.31 / 10.3 \%$ & $23.5 / 20.2 / 14.1 \%$ & $25.2 / 21.7 / 13.7 \%$ & $26.7 / 23.2 / 13.3 \%$ \\
\hline
\end{tabular}

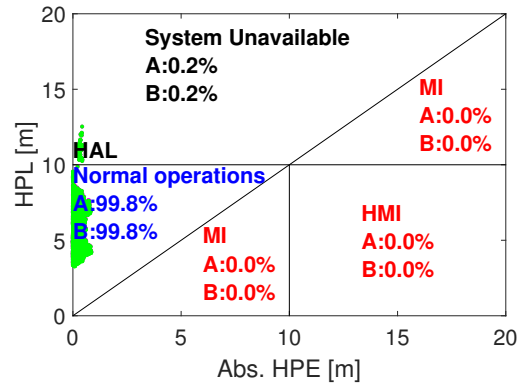

(a) Open-sky scenario

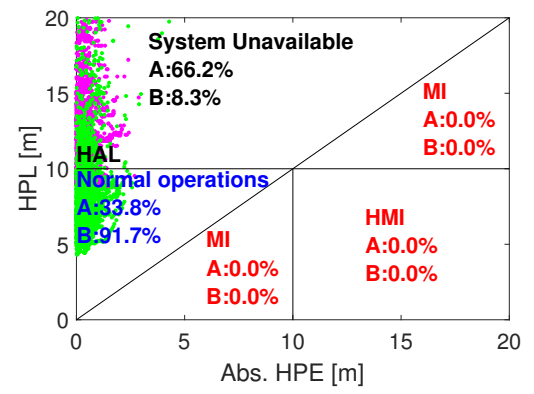

(b) Suburban scenario



(c) Urban scenario

Fig. 9 The Stanford ESA diagrams for the cases applying the $w_{A}^{s}$ (magenta dots) and $w_{B}^{s}$ (green dots) in (a) the open-sky scenario, (b) the suburban scenario and (c) the urban scenario showing the percentage of agreement in each category. The HAL, the $\mathrm{PHMI}_{\mathrm{HOR}}$ and the $N$ are set to $10 \mathrm{~m}, 10^{-5}$ and 900 epochs, respectively, for the plots. The x- and the y-axis are scaled to $20 \mathrm{~m}$ for purpose of visualisation. MI and HMI stand for the Misleading information and the Hazardously Misleading Information, respectively. A and B refer to Model A and B, respectively

models. MI stands for the Misleading Information with either $\mathrm{HPL}<\mathrm{HPE}<\mathrm{HAL}$ or $\mathrm{HAL}<\mathrm{HPL}<\mathrm{HPE}$, and HMI denotes the Hazardously Misleading Information having HPL $<$ HAL $<$ HPE. The system is unavailable when HAL $<$ HPL, and normal operation implies HPE $<$ HPL $<$ HAL. For purpose of visualisation the $\mathrm{x}-$ and the y-axis are scaled to $20 \mathrm{~m}$. While no significant differences are observed from the figure applying both weighting models in the open-sky scenario, applying $w_{B}^{s}$ (green dots) has improved the probability of the normal operation from about $35 \%$ to $90 \%$ in the suburban scenario, and from $0 \%$ to about $20 \%$ in the urban scenario. This is consistent with the significant reduction of the mean HPLs in the suburban and urban scenarios shown in Table 5 .

Since the integrity risk is application-dependent, more details of the integrity performance with respect to other possible values of $\mathrm{PHMI}_{\mathrm{HOR}}$ and smoothing windows are given in Table 6 . The values applying $w_{A}^{s}$ and $w_{B}^{s}$ are separated by ' $/$ ', and the values are given for $\mathrm{PHMI}_{\mathrm{HOR}}$ of $10^{-5}, 10^{-6}$ and $10^{-7}$. Results support the previous findings. While the integrity performance are similar applying both weighting models in the open-sky scenario, significant improvements can be observed applying $w_{B}^{s}$ in the suburban and urban scenarios for all the tested smoothing windows and $\mathrm{PHMI}_{\mathrm{HOR}}$, especially for the suburban scenario. Note that the results are used only to demonstrate the differences between the two weighting models, but are not attempted to verify the corresponding $\mathrm{PHMI}_{\mathrm{HOR}}$. 
Table 6 Integrity performance applying $w_{A}^{s}$ and $w_{B}^{s}$ for different measurement scenarios, smoothing windows and $\mathrm{PHMI}_{\mathrm{HOR}}$. The values applying $w_{A}^{s}$ and $w_{B}^{s}$ are separated by '/'

\begin{tabular}{|c|c|c|c|c|c|c|c|c|c|c|c|c|c|c|c|}
\hline & \multirow{2}{*}{\multicolumn{3}{|c|}{$\begin{array}{c}\text { Normal Operations [\%] } \\
\mathrm{HPE}<\mathrm{HPL}<\mathrm{HAL}\end{array}$}} & \multicolumn{6}{|c|}{ MI [\%] } & \multirow{2}{*}{\multicolumn{3}{|c|}{$\begin{array}{c}\text { HMI [\%] } \\
\text { HPL }<\text { HAL }<\text { HPE } \\
\end{array}$}} & \multirow{2}{*}{\multicolumn{3}{|c|}{$\begin{array}{c}\text { System unavailable [\%] } \\
\mathrm{HAL}<\mathrm{HPL}\end{array}$}} \\
\hline & & & & \multicolumn{3}{|c|}{ HPL $<$ HPE $<$ HAL } & \multicolumn{3}{|c|}{$\mathrm{HAL}<\mathrm{HPL}<\mathrm{HPE}$} & & & & & & \\
\hline${ }_{N}$ PHMI & $10^{-5}$ & $10^{-6}$ & $10^{-7}$ & $10^{-5}$ & $10^{-6}$ & $10^{-7}$ & $10^{-5}$ & $10^{-6}$ & $10^{-7}$ & $10^{-5}$ & $10^{-6}$ & $10^{-7}$ & $10^{-5}$ & $10^{-6}$ & $10^{-7}$ \\
\hline \multicolumn{16}{|c|}{ Open-sky scenario } \\
\hline 360 & $99.8 / 99.8$ & $99.5 / 99.5$ & $99.4 / 99.4$ & $0 / 0$ & $0 / 0$ & $0 / 0$ & $0 / 0$ & $0 / 0$ & $0 / 0$ & $0 / 0$ & $0 / 0$ & $0 / 0$ & $0.2 / 0.2$ & $0.5 / 0.5$ & $0.6 / 0.6$ \\
\hline 600 & $99.8 / 99.8$ & $99.5 / 99.5$ & $99.4 / 99.4$ & $0 / 0$ & $0 / 0$ & $0 / 0$ & $0 / 0$ & $0 / 0$ & $0 / 0$ & $0 / 0$ & $0 / 0$ & $0 / 0$ & $0.2 / 0.2$ & $0.5 / 0.5$ & $0.6 / 0.6$ \\
\hline 900 & $99.8 / 99.8$ & 99.5/99.5 & $99.4 / 99.4$ & $0 / 0$ & $0 / 0$ & $0 / 0$ & $0 / 0$ & $0 / 0$ & $0 / 0$ & $0 / 0$ & $0 / 0$ & $0 / 0$ & $0.2 / 0.2$ & $0.5 / 0.5$ & $0.6 / 0.6$ \\
\hline \multicolumn{16}{|c|}{ Suburban scenario } \\
\hline 360 & $38 / 89$ & $27 / 85$ & $9 / 79$ & $0 / 0$ & $0 / 0$ & $0 / 0$ & $0 / 0$ & $0 / 0$ & $0 / 0$ & $0 / 0$ & $0 / 0$ & $0 / 0$ & $62 / 11$ & $73 / 15$ & $91 / 21$ \\
\hline 600 & $33 / 91$ & $19 / 86$ & $2 / 81$ & $0 / 0$ & $0 / 0$ & $0 / 0$ & $0 / 0$ & $0 / 0$ & $0 / 0$ & $0 / 0$ & $0 / 0$ & $0 / 0$ & $67 / 9$ & $81 / 14$ & $98 / 19$ \\
\hline 900 & $34 / 92$ & $19 / 86$ & $1 / 81$ & $0 / 0$ & $0 / 0$ & $0 / 0$ & $0 / 0$ & $0 / 0$ & $0 / 0$ & $0 / 0$ & $0 / 0$ & $0 / 0$ & $66 / 8$ & $81 / 14$ & $99 / 19$ \\
\hline \multicolumn{16}{|c|}{ Urban scenario } \\
\hline 360 & $0 / 20$ & $0 / 16$ & $0 / 12$ & $0 / 0$ & $0 / 0$ & $0 / 0$ & $0.05 / 0$ & $0.05 / 0$ & $0.05 / 0$ & $0 / 0$ & $0 / 0$ & $0 / 0$ & $100 / 82$ & $100 / 84$ & $100 / 88$ \\
\hline 600 & $0 / 22$ & $0 / 17$ & $0 / 14$ & $0 / 0$ & $0 / 0$ & $0 / 0$ & $0.05 / 0$ & $0.05 / 0$ & $0.05 / 0$ & $0 / 0$ & $0 / 0$ & $0 / 0$ & $100 / 78$ & $100 / 83$ & $100 / 86$ \\
\hline 900 & $0 / 20$ & $0 / 16$ & $0 / 12$ & $0 / 0$ & $0 / 0$ & $0 / 0$ & $0.05 / 0$ & $0.05 / 0$ & $0.05 / 0$ & $0 / 0$ & $0 / 0$ & $0 / 0$ & $100 / 80$ & $100 / 84$ & $100 / 88$ \\
\hline
\end{tabular}

\section{Conclusion}

The second-generation SBAS test-bed was initiated in 2017 in Australia and New Zealand. The SBAS DFMC message broadcast from the GEO satellite supports the positioning service based on dual-frequency GPS and Galileo signals. Having the SBAS DFMC positioning and IM procedures strictly defined for aeronautical applications (ED-259, EUROCAE 2019), the different and more complicated measurement environments on the ground require a detailed study of the weighting models for the combined noise and multipath effects different from that applied for aviation. It is shown that in the urban environment, e.g., the large multipath effects may not necessarily only occur at low elevation angles, and the frequent filter re-initialisations due to cycle slips and data gaps also lead to large residuals within short filtering smoothing time. A simple elevation-dependent weighting function might thus not be enough to describe the combined noise and multipath behaviours in complicated measurement environments. Therefore, a new weighting model considering not only the elevation angles, but also the SNRs and the smoothing time is proposed and investigated for different smoothing windows and different environments, i.e., the open-sky, the suburban and the urban scenarios. By varying the coefficients of the new weighting model, different impacts of these influence factors can be taken into consideration in the positioning process.

Making use of the real data collected in different environments for the signal analysis, the effects of the traditional elevation-dependent weighting model and the proposed new weighting model were compared. It was found that the elevation-dependent weighting model is sufficient for the open-sky scenario, while in the suburban and urban scenarios, the new weighting model considering the SNRs and the smoothing time can lead to a better match of the empirical distribution for the combined weighted and normalised noise and multipath to the standard normal distribution. Applying the new weighting model, the overbounding mean values and standard deviations of the EMCs are significantly reduced in the suburban and urban scenarios, while similar results were obtained applying both weighting models in the open-sky scenario. In the challenging urban environment, the weighting model related to the SNRs and the smoothing time is suggested to be used for the DFMC SBAS positioning and integrity monitoring. For GPS measurements, the SNR coefficients $\tilde{m}_{k}$ could range from 20 to $30 \mathrm{dBHz}$, and the smoothing coefficient $\beta$ could range from 0.15 to 0.25 . For Galileo measurements, the SNR coefficients $\tilde{m}_{k}$ could range from 50 to $60 \mathrm{dBHz}$, and the smoothing coefficient $\beta$ could be set to 0.05 .

Using $1 \mathrm{~Hz}$ real data for the SBAS DFMC positioning, applying the new weighting model in the suburban and urban scenarios is found to be able to significantly reduce the mean HPLs in the suburban and urban scenarios, leading to improvement in the IM availability. Improvements in the mean absolute HPEs are also observed in the urban scenario. In contrast, the results in the open-sky scenario are similar applying both models. This, again, supports our recommendation to use the elevation-dependent model in the open-sky scenario, and the new weighting model for ground-based applications in the suburban and urban scenarios. 


\section{Acknowledgements}

The authors would like to thank the GMV company (Spain) for providing the SBAS messages on the test days. The authors appreciate the support of Septentrio for providing the receiver for the station UWA0 and the University of Western Australia for hosting this station. The data of UWA0 was obtained from GNSS Research Centre, Curtin University through http://saegnss2.curtin.edu/ldc/. The authors would also like to thank Dr. Davide Imparato for collecting the data in Sydney and Wollongong, and SmartNet for providing the data of the stations FTDN and GONG. Thanks are also given to the Transport for New South Wales (NSW) for realizing the experiments at NSW. This work is funded by the Australian Research Council Discovery Project: Trustworthy Positioning for Next Generation Intelligent Transport Systems, Project ID: DP170103341.

\section{Author contribution statement}

Author contributions: K.W. and A.E. designed the research; K.W. performed the research and analyzed the data; K.W. wrote the paper, A.E., C.R., J.W. revised the the paper.

\section{Data availability statement}

The data of the stations UWA0 were obtained from the GNSS Research Centre, Curtin University through http://saegnss2.curtin.edu/ldc/. The data of the kinematic experiments were collected by the GNSS group of Curtin, and can be provided for internal use upon request.

\section{References}

ARAIM Technical Subgroup (2016) EU-U.S. cooperation on satellite navigation, Working group C ARAIM technical subgroup, Milestone 3 report, Final version, February 25th, 2016

Barrios J, Caro J, Calle JD, Carbonell E, Pericacho JG, Fernández G, Esteban VM, Fernández MA, Bravo F, Torres B, Calabrese A, Diaz A, Rodríguez I, Laínez MD, Romay MM, Jackson R, Reddan PE, Bunce D, Soddu C (2018) Update on Australia and New Zealand DFMC SBAS and PPP System Results. In Proc. ION GNSS+ 2018, Miami, Florida, September 24-28, 2018, pp. 1038-1067. doi:10.33012/2018.15932

Blanch J, Walter T, Enge P (2013) Optimal positioning for advanced Raim. NAVIGATION: Journal of The Institute of Navigation, 60(4):279-289. doi:10.1002/navi.49

Blanch J, Walter T, Enge P, Lee Y, Pervan B, Rippl M, Spletter A (2012) Advanced RAIM user algorithm description: Integrity support message processing, fault detection, exclusion, and protection level calculation. In Proc. of ION GNSS, Nashville, TN, September 2012, pp. 2828-2849

Choy S, Kuckartz J, Dempster AG, Rizos C, Higgins M (2017) GNSS satellite-based augmentation systems for Australia. GPS Solutions 21(3):835-848. doi:10.1007/s10291-016-0569-2

DeCleene B (2000) Defining pseudorange integrity - Overbounding. In Proc. ION GPS 2000, Salt Lake City, UT, September 2000, pp.1916-1924.

El-Mowafy A (2015) Estimation of multi-constellation GNSS observation stochastic properties using single receiver single satellite data validation method. Survey Review 47(341):99-108. doi:10.1179/ 1752270614 Y.0000000100

El-Mowafy A (2017) Advanced receiver autonomous integrity monitoring using triple frequency data with a focus on treatment of biases. Advances in Space Research, 59(8):2148-2157. doi:10.1016/j.asr.2017.01.037

El-Mowafy A, Kubo N (2017) Integrity monitoring of vehicle positioning in urban environment using RTKGNSS, IMU and speedometer. Measurement Science and Technology, 28:055102. doi:10.1088/1361-6501/ aa5c66

El-Mowafy A, Kubo N (2018) Integrity monitoring for positioning of intelligent transport systems using integrated RTK-GNSS, IMU and vehicle odometer. IET Intelligent Transport Systems 12(8):901-908. doi:10.1049/iet-its.2018.0106

Euler HJ, Goad CC (1991) On optimal filtering of GPS dual frequency observations without using orbit information. Bulletin géodésique, 65(2): 130-143. doi:10.1007/BF00806368 
EUROCAE (2019) Minimum operational performance standard for Galileo/global positioning system/satellite-based augmentation system airborne equipment. The European Organisation for civil aviation equipment, ED-259, February 2019

FrontierSI (2017) Technical specifications document for satellite-based augmentation system (SBAS) testbed. Revision 5, November 10, 2017

Grunwald G, Bakula M, Ciećko C (2016) Study of EGNOS accuracy and integrity in eastern Poland. The Aeronautical Journal, 120(1230):1275-1290. doi:10.1017/aer.2016.66

Gunning K, Blanch J, Walter T, de Groot L, Norman L (2018) Design and evaluation of integrity algorithms for PPP in kinematic applications. In Proc. ION GNSS+ 2018, Miami, Florida, September 2018, pp. 1910-1939

Hartinger H, Brunner FK (1999) Variances of GPS phase observations: The SIGMA- $\epsilon$ Model. GPS Solutions, 2(4):35-43. doi:10.1007/PL00012765

Hatch R (1982) The synergism of GPS code and carrier measurements. In Proc. the third International Geodetic Symposium on Satellite Doppler Positioning, Vol. 2, Las Cruces, New Mexico, 1982, pp. 12131231.

Hoque MM, Jakowski N (2012) Ionospheric propagation effects on GNSS signals and new correction approaches. In Book: Global Navigation Satellite Systems: Signal, Theory and Applications, Jin S, IntechOpen. doi:10.5772/30090

Le AQ, Teunissen PJG (2008) Optimal recursive least-squares filtering of GPS pseudorange measurements. In: Xu P, Liu J, Dermanis A (eds) VI Hotine-Marussi Symposium on Theoretical and Computational Geodesy. International Association of Geodesy Symposia, vol 132. Springer, Berlin, Heidelberg. doi: 10.1007/978-3-540-74584-6_26

Lee YC (1986) Analysis of range and position comparison methods as a means to provide GPS integrity in the user receiver. In Proc. the 42nd Annual Meeting of the Institute of Navigation, Seattle, Washington, June 1986, pp. 1-4

Malkin Z (2008) Influence of the elevation-dependent weighting on parameter estimates: A case of CONT05. In Proc. the 5th IVS General Meeting, St. Petersburg, Russia, March 2-6, 2008, pp. 178-182.

Nadarajah N, Khodabandeh A, Teunissen PJG (2016) Assessing the IRNSS L5-signal in combination with GPS, Galileo, and QZSS L5/E5a-signals for positioning and navigation. GPS Solutions, 20(2):289-297. doi:10.1007/s10291-015-0450-8

Parkinson BW, Axelrad P (1988) Autonomous GPS integrity monitoring using the pseudorange residual. NAVIGATION: Journal of the Institute of Navigation, 35(2):225-274. doi:10.1002/j.2161-4296.1988. tb00955.x

Rife J, Pullen S, Enge P, Pervan B (2006) Paired overbounding for nonideal LAAS and WAAS error distributions. IEEE Transactions on Aerospace and Electronic Systems, 42(4):1386-1395. doi:10.1109/ TAES.2006.314579

Rife J, Walter T, Blanch J (2004) Overbounding SBAS and GBAS error distributions with excess-mass functions. In Proc. the 2004 International Symposium on GNSS/GPS, Sydney, Australia, 6-8 December, 2004.

RINEX 3.03 (2015) RINEX, The receiver independent exchange format, Version 3.03. International GNSS Service (IGS), RINEX Working Group and Radio Technical Commission for Maritime Services Special Committee 104 (RTCM-SC104), July 14, 2015.

RTCA (2016) RTCA DO-229E, Minimum operational performance standards for global positioning system/satellite-based augmentation system airborne equipment, 2016.

Tay S, Marais J (2013) Weighting models for GPS pseudorange observations for land transportation in urban canyons. 6th European Workshop on GNSS Signals and Signal Processing, Dec 2013, Germany. 4p. hal-00942180

Teunissen PJG, de Bakker PF (2012) Next generation GNSS single receiver cycle slip reliability. In: Sneeuw N, Novák P, Crespi M, Sansò F (eds) VII Hotine-Marussi Symposium on Mathematical Geodesy. International Association of Geodesy Symposia, vol 137, 159-164. Springer, Berlin, Heidelberg

Tossaint M, Samson J, Toran F, Ventura-Traveset J, Sanz J, Hernandez-Pajares M, Juan JM (2006) The Stanford - ESA Integrity Diagram: Focusing on SBAS Integrity. In Proc. ION GNSS 2006, Fort Worth, TX, September 2006, pp. 894-905

Walter T, Blanch J, Enge P (2010a) Vertical protection level equations for dual frequency SBAS. In Proc. of ION GNSS 2010, Portland, OR, September 2010, pp. 2031-2041

Walter T, Blanch J, Enge P (2010b) Coverage improvement for dual frequency SBAS. In Proc. the 2010 International Technical Meeting of The Institute of Navigation, San Diego, CA, January 2010, pp. 344- 
353.

Wang K, El-Mowafy A, Rizos C, Wang J (2020) Integrity Monitoring for Horizontal RTK Positioning: New Weighting Model and Overbounding CDF in Open-Sky and Suburban Scenarios. Remote Sensing, 12(7), 1173. doi: $10.3390 / \mathrm{rs} 12071173$

Wanner B, DeCleene B, Nelthropp DA, Gordon S (2008) Wide area augmentation system vertical accuracy assessment in support of LPV200 requirements. NAVIGATION: Journal of the Institute of Navigation, 55(3):191-203. doi:10.1002/j.2161-4296.2008.tb00429.x

Wieser A and Brunner FK (2000) An extended weight model for GPS phase observations. Earth, Planets and Space, 52:777-782. doi:10.1186/BF03352281

Zhou W, Liu L, Huang L, Yao Y, Chen J, Li S (2019) A new GPS SNR-based combination approach for land surface snow depth monitoring. Scientific Reports 9, No. 3814. doi:10.1038/s41598-019-40456-2

\section{A Derivation of $w_{B}^{s}$}

Assuming that the signal standard deviations $\sigma_{0, k \text {, raw }}$ are equal on both frequencies, and considering only the SNRs in the weighting model, the variance of the IF slant raw observations can be formulated as:

$$
\begin{aligned}
\sigma_{s, \text { raw }}^{2} & =\frac{\sigma_{0, k, \text { raw }}^{2} \times f_{u}^{4} \times\left(1+m_{u} \times 10^{-0.1 \times S_{u}^{s}}\right)}{\left(f_{u}^{2}-f_{v}^{2}\right)^{2}}+\frac{\sigma_{0, k, \text { raw }}^{2} \times f_{v}^{4} \times\left(1+m_{v} \times 10^{\left.-0.1 \times S_{v}^{s}\right)}\right.}{\left(f_{u}^{2}-f_{v}^{2}\right)^{2}} \\
& =\underbrace{\frac{\sigma_{0, k, \text { raw }}^{2} \times\left(f_{u}^{4}+f_{v}^{4}\right)}{\left(f_{u}^{2}-f_{v}^{2}\right)^{2}}}_{\sigma_{0, \text { raw }}^{2}} \times\left(1+\frac{f_{u}^{4}}{f_{u}^{4}+f_{v}^{4}} \times m_{u} \times 10^{-0.1 \times S_{u}^{s}}+\frac{f_{v}^{4}}{f_{u}^{4}+f_{v}^{4}} \times m_{v} \times 10^{-0.1 \times S_{v}^{s}}\right)
\end{aligned}
$$

Considering the elevation angles in addition, $\sigma_{s, \text { raw }}^{2}$ can be expanded to

$$
\sigma_{s, \text { raw }}^{2}=\sigma_{0, \text { raw }}^{2} \times\left(1+\alpha \times \exp \left(-\frac{\theta^{s}}{10^{\circ}}\right)\right)^{2} \times\left(1+\frac{f_{u}^{4}}{f_{u}^{4}+f_{v}^{4}} \times m_{u} \times 10^{-0.1 \times S_{u}^{s}}+\frac{f_{v}^{4}}{f_{u}^{4}+f_{v}^{4}} \times m_{v} \times 10^{-0.1 \times S_{v}^{s}}\right)
$$

Based on Eq. A.2, the variance of the smoothed observations can be derived with:

$$
\begin{aligned}
\sigma_{s}^{2}\left(t_{i}\right) & =\frac{\sum_{j=i-n+1}^{i} \sigma_{s, \text { raw }}^{2}\left(t_{j}\right)}{n^{2}} \\
& =\frac{\sigma_{0, \text { raw }}^{2} \times \sum_{j=i-n+1}^{i}\left(1+\alpha \times \exp \left(-\frac{\theta^{s}\left(t_{j}\right)}{10^{\circ}}\right)\right)^{2} \times\left(1+\frac{f_{u}^{4}}{f_{u}^{4}+f_{v}^{4}} \times m_{u} \times 10^{-0.1 \times S_{u}^{s}\left(t_{j}\right)}+\frac{f_{v}^{4}}{f_{u}^{4}+f_{v}^{4}} \times m_{v} \times 10^{-0.1 \times S_{v}^{s}\left(t_{j}\right)}\right)}{n^{2}} \\
& \approx \frac{\sigma_{0, \text { raw }}^{2} \times\left(1+\alpha \times \exp \left(-\frac{\theta^{s}\left(t_{i}\right)}{10^{\circ}}\right)\right)^{2} \times \sum_{j=i-n+1}^{i}\left(1+\frac{f_{u}^{4}}{f_{u}^{4}+f_{v}^{4}} \times m_{u} \times 10^{-0.1 \times S_{u}^{s}\left(t_{j}\right)}+\frac{f_{v}^{4}}{f_{u}^{4}+f_{v}^{4}} \times m_{v} \times 10^{-0.1 \times S_{v}^{s}\left(t_{j}\right)}\right)}{n^{2}} \\
& =\frac{N \times \sigma_{0}^{2} \times\left(1+\alpha \times \exp \left(-\frac{\theta^{s}\left(t_{i}\right)}{10^{\circ}}\right)\right)^{2} \times\left(n+n \times \frac{f_{u}^{4}}{f_{u}^{4}+f_{v}^{4}} \times m_{u} \times 10^{-0.1 \times \bar{S}_{u}^{s}\left(t_{i}\right)}+n \times \frac{f_{v}^{4}}{f_{u}^{4}+f_{v}^{4}} \times m_{v} \times 10^{-0.1 \times \bar{S}_{v}^{s}\left(t_{i}\right)}\right)}{n^{2} \times \sigma_{0}^{2} \times \frac{1}{w_{A}^{s}\left(\theta^{s}\left(t_{i}\right)\right)} \times\left(1+\frac{f_{u}^{4}}{f_{u}^{4}+f_{v}^{4}} \times m_{u} \times 10^{-0.1 \times \bar{S}_{u}^{s}\left(t_{i}\right)}+\frac{f_{v}^{4}}{f_{u}^{4}+f_{v}^{4}} \times m_{v} \times 10^{-0.1 \times \bar{S}_{v}^{s}\left(t_{i}\right)}\right)}
\end{aligned}
$$

where it is assumed that $w_{A}^{s}\left(\theta^{s}\left(t_{i-n+1}\right)\right) \approx \cdots \approx w_{A}^{s}\left(\theta^{s}\left(t_{i}\right)\right)$. Based on Eq. A.3, the weighting model $w_{B}^{s}$ at $t_{i}$ can be approximated as shown in Eq. 13:

$w_{B}^{s}\left(\theta^{s}\left(t_{i}\right), \bar{S}_{k}^{s}\left(t_{i}\right), n\right)=\frac{\sigma_{0}^{2}}{\sigma_{s}^{2}\left(t_{i}\right)}=w_{A}^{s}\left(\theta^{s}\left(t_{i}\right)\right) \times \frac{1}{1+\frac{N-n}{n}} \times \frac{1}{\left(1+\frac{f_{u}^{4}}{f_{u}^{4}+f_{v}^{4}} \times m_{u} \times 10^{-0.1 \times \bar{S}_{u}^{s}\left(t_{i}\right)}+\frac{f_{v}^{4}}{f_{u}^{4}+f_{v}^{4}} \times m_{v} \times 10^{-0.1 \times \bar{S}_{v}^{s}\left(t_{i}\right)}\right)}$ 